\title{
COMPOUND GROUP EXTENSIONS. III
}

\author{
BX \\ ROBERT L. TAYLOR
}

1. Introduction $\left({ }^{1}\right)$. Let $\phi: K \rightarrow G$ be a normal homomorphism, with $X=\phi^{-1}(0), N=\phi(K), Q=G / N$, as in [CGE I]. Any continuation $\phi: E \rightarrow G$ of $\phi: K \rightarrow G$ induces several different "operations," which determine each other in accordance with the following scheme:

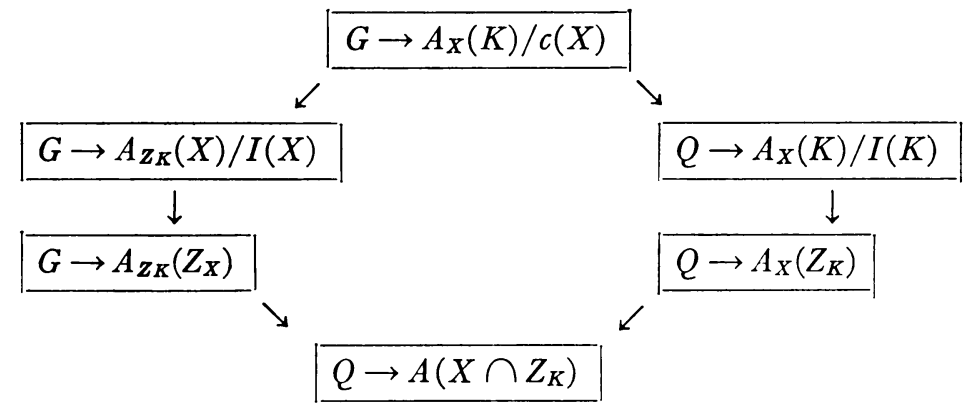

where $A_{L}(M)$ denotes the group of all automorphisms of $M$ which map $L \cap M$ onto itself, and $Z_{M}$ denotes the center of $M$.

The role of $H^{n}\left(Q, X \cap Z_{K}\right)$ in [CGE I] results from the adoption of $G \rightarrow A_{X}(K) / c(X)$ as the type of "operation" to be held fixed. In [CGE IV], $G \rightarrow A(X) / I(X)$ is held fixed, with the result that continuations are classified in terms of the relative cohomology groups $H^{n}\left(G, N ; Z_{X}\right)$. In the present paper we explore the effects of adopting $Q \rightarrow A_{X}(K) / I(K)$ as the fixed "operation." We show that in this situation the relevant cohomology groups, as far as continuations are concerned, are the groups $H^{n}(\phi)$ of the mapping $C^{n}\left(Q, Z_{K}\right) \rightarrow C^{n}\left(Q, Z_{N}\right)$. Specifically, we find an obstruction in $H^{3}(\phi)$ to the

Received by the editors June 16, 1953 and, in revised form, September 15, 1954.

(1) Rudimentary portions of this paper appeared in [CFB]; the remainder was written while the author was partially supported by the Office of Scientific Research (ARDC). The notation and terminology of [CGE I] and [CGE II] will be extensively used throughout. Symbols such as [CFB] and [CGE I] refer to the bibliography at the end of the paper.

In addition to the notational conventions of [CGE I], we call the reader's attention to our use of $\rho$ (mentioned in $\S 4$ ) for the natural mapping of any group of cocycles onto the corresponding cohomology group; the cochain complex in question is to be identified from the context. The proofs of (9.11) and (9.12) are examples of this usage.

We take this occasion to point out three misprints. In [CGE I, p. 111], diagram (2.6), of the two vertical arrows labelled $C_{N}$, the one on the left should be $c_{K}$, while the one on the right should be $c_{N}$. In [CGE II, p. 307], the seventh line below the diagram should read $R_{0}=S_{0}=R_{4}$ $=S_{\mathbf{4}}=0$. 
existence of a continuation of $\phi: K \rightarrow G$ inducing a given $Q \rightarrow A_{X}(K) / I(K)$, and we show that a certain modification $\widetilde{H}^{2}(\phi)$ of $H^{2}(\phi)$ operates simply transitively on the set of all isomorphism-classes of such continuations. $H^{2}(\phi)$ itself operates simply transitively on the set of all (appropriately defined) "similarity classes" of such continuations.

We are interested in classifying not only the continuations, but also the modular structures which induce a given $Q \rightarrow A_{X}(K) / I(K)$. Moreover we wish to study these two questions in relation to each other, and to the classification of the extensions of the $Q$-kernels $Q \rightarrow A_{\boldsymbol{X}}(K) / I(K)$ and $Q \rightarrow A(N) / I(N)$. For such purposes it helps to regard the extension $(G, \mu)$ of $Q$ by $N$ as a variable rather than a constant. Consequently, we shall begin not with a normal homomorphism $\phi: K \rightarrow G$, but merely with two groups $Q$ and $K$, a normal subgroup $X$ of $K$, and an arbitrary homomorphism $\tau: Q \rightarrow A_{X}(K) / I(K) . N$ will denote $K / X$, and $\phi: K \rightarrow N$ the natural homomorphism. Recalling from [CGE I] the induced homomorphism $\phi_{0}: A_{X}(K) / I(K) \rightarrow A(N) / I(N)$, we obtain a "derived" $Q$-kernel $\phi_{0} \tau: Q \rightarrow A(N) / I(N)$. We now redefine a "continuation" as an extension

$$
0 \rightarrow N \rightarrow G \stackrel{\mu}{\rightarrow} Q \rightarrow 0
$$

of the $Q$-kernel $\phi_{0} \tau: Q \rightarrow A(N) / I(N)$, together with a continuation $\phi: E \rightarrow G$ of $\phi: K \rightarrow G$ in the earlier sense of [CGE I], which induces $\tau: Q \rightarrow A_{X}(K) / I(K)$. In the definition of "isomorphism" of continuations, we insist that the two extensions of the $Q$-kernel $\phi_{0} \tau: Q \rightarrow A(N) / I(N)$ be not merely isomorphic but identical. (The precise definitions in the correct logical order are given in $\$ 3$.)

The above results concerning $H^{n}(\phi)$ are now formulated as follows: $\widetilde{H}^{2}(\phi)$ operates simply, but not transitively, on the set of all isomorphism-classes of continuations, the orbits being the nonempty counter-images of individual extensions of $\phi_{0} \tau: Q \rightarrow A(N) / I(N)$ under the natural mapping

$$
\text { (continuations) } \rightarrow\left(\text { extensions of the } Q \text {-kernel } \phi_{0} \tau\right) \text {; }
$$

in place of an individual "obstruction element" of $H^{3}(\phi)$ we now have a "natural mapping" of the set of all extensions of the $Q$-kernel $\phi_{0} \tau$ into $H^{3}(\phi)$, such that the sequence

$$
\text { (continuations) } \rightarrow\left(\text { extensions of the } Q \text {-kernel } \phi_{0} \tau\right) \stackrel{\chi}{\rightarrow} H^{3}(\phi)
$$

is "exact." Furthermore, we are now able to formulate and establish supplementary facts such as the following: the mapping $\chi$ : (extensions of $\left.\phi_{0} \tau\right) \rightarrow H^{3}(\phi)$ is "equivariant" relative to the natural homomorphism $H^{2}\left(Q, Z_{N}\right) \rightarrow H^{3}(\phi)$; the image in $H^{3}(\phi)$ of the set of all extensions of $\phi_{0} \tau$ (whether empty or not) is equal to the counter-image, under the natural homomorphism $H^{3}(\phi)$ $\rightarrow H^{3}\left(Q, Z_{K}\right)$, of the Eilenberg-MacLane obstruction [CTAG II] of the $Q$ kernel $\tau: Q \rightarrow A_{X}(K) / I(K)$. 
Let us now define a "modular expansion" as an extension (1.2) of the $Q$-kernel $\phi_{0} \tau: Q \rightarrow A(N) / I(N)$ together with a modular structure

$$
\theta: G \rightarrow A_{X}(K) / c(X)
$$

on $\phi: K \rightarrow G$ which induces $\tau: Q \rightarrow A_{X}(K) / I(K)$, i.e. which satisfies

$$
\omega \theta=\tau \mu,
$$

where $\omega: A_{X}(K) / c(X) \rightarrow A_{X}(K) / I(K)$ is the natural homomorphism. This concept is somewhat similar to that of a "related crossed module," as defined in [APC]. However, the results of [APC] are not directly applicable here, for three reasons: (1) in [APC] it is assumed that $X \subset Z_{K}$, a restriction which is no longer needed when "crossed modules" are replaced by "pseudomodules"; (2) in [APC], the homomorphism $\mu: G \rightarrow Q$ is not considered part of the structure-such a homomorphism is merely required to exist; hence the mapping (modular expansions) $\rightarrow$ (related crossed or pseudo-modules) is onto but many-to-one; (3) the results of [APC] are partly false (a counterexample is given in [QKO II], and the results are corrected in [QKO I]).

Nevertheless, [APC] does indicate that the kernel of $H^{2}\left(Q, \phi Z_{K}\right)$ $\rightarrow H^{2}\left(Q, Z_{N}\right)$ operates simply transitively on the set of all isomorphism-classes of modular expansions which determine a given isomorphism-class of extensions of the $Q$-kernel $\phi_{0} \tau: Q \rightarrow A(N) / I(N)$, and that the image of $H^{2}\left(Q, \phi Z_{K}\right)$ $\rightarrow H^{2}\left(Q, Z_{N}\right)$ operates simply transitively on the set of all isomorphism-classes of extensions of $\phi_{0} \tau: Q \rightarrow A(N) / I(N)$ which arise from modular expansions. This suggests, but does not prove, that $H^{2}\left(Q, \phi Z_{K}\right)$ operates simply transitively (in a natural way) on the set of all isomorphism-classes of modular expansions. We show that this is indeed the case, and that both this result and the criterion in [APC] for the existence of a modular expansion (minus the restriction $X \subset Z_{K}$ ) are in fact special cases, via [CGE II], of the main results of $[\mathrm{CGE} \mathrm{I}]$.

The main results of the present paper are all stated in $\$ 5$ (using terminology and notation developed in $\$ \S 2-4)$. They include all of the above, together with certain other operations of various groups on certain sets of "continuations" and "modular expansions," related by mappings which are "equivariant" in the sense of $\$ 2$. Proofs of the major portions of these results are given in Part II ( $\$ 7-10)$. Proofs of the remaining portions, involving for the most part groups of cocycles rather than of cohomology classes, are given in Part III $(\$ \$ 11-16)$.

The present study was originally undertaken in order to clarify the following phenomena. If $\phi: K \rightarrow G, \theta: G \rightarrow A_{X}(K)$ is a crossed module in which $K$ is abelian, then the exact sequence

$$
0 \rightarrow X \rightarrow K \stackrel{\phi}{\rightarrow} N \rightarrow 0
$$

of operator homomorphisms rel. $Q$ induces an exact sequence of cohomology groups 


$$
\cdots \rightarrow H^{n}(Q, X) \rightarrow H^{n}(Q, K) \stackrel{\phi_{*}}{\rightarrow} H^{n}(Q, N) \stackrel{\nabla}{\rightarrow} H^{n+1}(Q, X) \rightarrow \cdots,
$$

and the obstruction of the crossed module is precisely $\nabla(\gamma) \in H^{3}(Q, X)$, where $\gamma \in H^{2}(Q, N)$ is the element corresponding to the group extension (1.2) of $Q$ by $N$. Hence the crossed module is extendible if and only if

$$
\gamma \in \phi_{*} H^{2}(Q, K) \text {. }
$$

In attempting to generalize the criterion (1.8) to the nonabelian case, we must replace $\gamma \in H^{2}(Q, N)$ by $\gamma \in \bar{H}^{2}\left(Q, N, \phi_{0} \tau\right)$ [CGE I, §5], where $\tau: Q$ $\rightarrow A_{X}(K) / I(K)$ is induced by the given modular structure $\theta: G \rightarrow A_{X}(K)$. Now $\phi: K \rightarrow N$ induces $\phi_{*}: \bar{H}^{2}(Q, K, \tau) \rightarrow H^{2}\left(Q, N, \phi_{0} \tau\right)$; hence we might try replacing (1.8) by

$$
\gamma \in \phi_{*} \bar{H}^{2}(Q, K, \tau) .
$$

This condition is still necessary for the extendibility of the crossed module, but it is no longer sufficient (actual counter-examples can be constructed). (1.9) is a necessary and sufficient condition for the existence of a continuation of $\phi: K \rightarrow G$ which induces $\tau: Q \rightarrow A_{X}(K) / I(K)$; but the modular structure induced by such a continuation need not coincide with the given $\theta$.

However, it turns out that (1.9) suffices for the extendibility of the crossed module in the situation of [CNG], i.e. when all groups are topological, $N$ is the component of the identity in $G$, and $K$ is a connected covering of $N$. More generally, condition (1.9) suffices (for pseudo-modules as well as crossed modules) whenever, in the notation of $\$ 4$, the "iterated connecting homomorphism"

$$
\nabla_{1} \nabla_{2}: H^{1}\left(Q, Z_{N} / \phi Z_{K}\right) \rightarrow H^{3}\left(Q, X \cap Z_{K}\right)
$$

is zero. This state of affairs is explained by the results of $\S 5$, which imply, as immediate corollaries:

(a) If (1.10) is zero, then any two modular structures on $\phi: K \rightarrow G$ which induce the same $\tau: Q \rightarrow A_{X}(K) / I(K)$ have the same obstruction; hence if one such is extendible, so are all others.

(b) If $\nabla_{2}: H^{1}\left(Q, Z_{N} / \phi Z_{K}\right) \rightarrow H^{2}\left(Q, \phi Z_{K}\right)$ is zero, then any two modular structures on $\phi: K \rightarrow G$ which induce the same $\tau: Q \rightarrow A_{X}(K) / I(K)$ are "congruent" $(\$ 3)$, and hence, a fortiori, have the same obstruction.

(c) If $\nabla_{2} \rho: Z^{1}\left(Q, Z_{N} / \phi Z_{K}\right) \rightarrow H^{2}\left(Q, \phi Z_{K}\right)$ has kernel zero, then any two congruent modular structures are equal. Combining this with (b): If

$$
Z^{1}\left(Q, Z_{N} / \phi Z_{K}\right)=0
$$

then any two modular structures on $\phi: K \rightarrow G$ which induce the same $\tau: Q$ $\rightarrow A_{X}(K) / I(K)$ are equal.

(d) If $\left(\alpha \pi_{N}\right)_{*}: H^{3}(\phi) \rightarrow H^{2}\left(Q, Z_{N} / \phi Z_{K}\right)$ is zero, then for every extension (1.2) of the $Q$-kernel $\phi_{0} \tau: Q \rightarrow A(N) / I(N)$, there exists a modular structure on $\phi: K \rightarrow G$ which induces $\tau: Q \rightarrow A_{X}(K) / I(K)$. 
In particular, if $\phi Z_{K}=Z_{N}$, then for every $(G, \mu)$ and $\tau$, there exists one and only one modular structure. Cf. [CNG, Proposition (6.2)].

In a sense, the best generalization to arbitrary pseudo-modules of the abelian formula $\chi(\phi, \theta)=\nabla(\gamma)$ [mentioned in connection with (1.8)] is the statement, included in Theorem 5.3, that $\chi: \mathrm{P} \rightarrow H^{3}\left(Q, X \cap Z_{K}\right)$ is equivariant relative to $\nabla_{1}: H^{2}\left(Q, \phi Z_{K}\right) \rightarrow H^{3}\left(Q, X \cap Z_{K}\right)$, i.e. $\chi(h+p)=\nabla_{1} h+\chi p$. The abelian formula is easily derived as a special case of this.

\section{PART I}

2. Equivariant diagrams. Let the additive group $A$ act additively as a group of left operators on the set $X$; let the additive group $B$ act similarly on the set $Y$; let $f: X \rightarrow Y$ be a (set-theoretic) mapping, and $\alpha: A \rightarrow B$ a homomorphism. We say that $f$ is equivariant rel. $\alpha$ if

$$
f(a+x)=\alpha(a)+f(x)
$$

for all $a \in A$ and all $x \in X$. If the formula

$$
f(a+x)=-\alpha(a)+f(x)
$$

holds in place of (2.1), we say that $f$ is antivariant rel. $\alpha$. If we assert that $f: X \rightarrow Y$ is equivariant or antivariant rel. $\alpha: A \rightarrow B$, and it happens that $Y=B$, it is to be understood that the operation of $B$ on itself is left translation.

A diagram of set-theoretic mappings

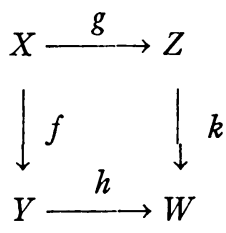

will be called equivariant relative to the diagram of groups and homomorphisms

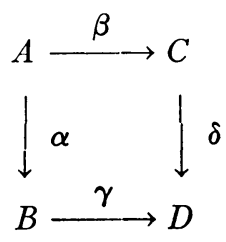

if each group in (2.4) operates on the correspondingly placed set in (2.3), and each mapping in (2.3) is equivariant relative to the corresponding homomorphism in (2.4). The same terminology will be used for more complicated pairs of diagrams, provided the set-diagram and the group-diagram have the same format. 
An operation of $A$ on $X$ is called simple if $[a \in A$ and there exists $x \in X$ such that $a+x=x]$ implies $[a=0]$; transitive if for every pair $\left(x, x^{\prime}\right) \in X \times X$ there exists $a \in A$ such that $a+x=x^{\prime}$; simply transitive if it is simple and transitive. The orbits in $X$ under $A$ are the equivalence classes of $X$ determined by the relation [ $x$ is equivalent to $x^{\prime}$ if there exists $a \in A$ such that $a+x=x^{\prime}$ ]. It follows from standard conventions that every group operates simply transitively on the empty set, and that the empty set is never counted as an equivalence class.

If $f: X \rightarrow Y$ and $g: X \rightarrow Z$ are mappings, $f \times g: X \rightarrow Y \times Z$ will denote the "cartesian product" of $f$ and $g$, defined by $(f \times g)(x)=\left(f x,{ }^{*} g x\right)$. If the mappings are indicated simply by the symbols $X \rightarrow Y$ and $X \rightarrow Z$ (without symbols such as $f, g), X \rightarrow Y \times Z$ will denote the cartesian product of $X \rightarrow Y$ and $X \rightarrow Z$.

We call the diagram (2.3) regular if, for all $(y, z) \in Y \times Z:(y, z) \in(f \times g)(X)$ if and only if $h(y)=k(z)$. Every regular diagram is commutative, but not conversely. We call (2.3) strict if $f \times g: X \rightarrow Y \times Z$ is 1-1 into; strictly regular if it is strict and regular.

A sequence of mappings $X \rightarrow^{f} Y \rightarrow^{0} A$, where $X$ and $Y$ are sets and $A$ is a group, will be called exact if $f(X)=g^{-1}(0)$.

If $A$ operates simply on $X$, and $x, x^{\prime}$ are elements of the same orbit in $X$ under $A$, then $x-x^{\prime}$ will denote the element $a \in A$ such that $a+x^{\prime}=x$.

Let $f: X \rightarrow Y$ be a set-theoretic mapping. By the fibers of $f: X \rightarrow Y$ we mean the equivalence classes of $X$ determined by the relation [ $x$ is equivalent to $x^{\prime}$ if $f(x)=f\left(x^{\prime}\right)$ ]. In other words, the fibers of a mapping are the nonempty counter-images of individual points.

The following propositions are easily verified:

(2.5) Let $f: X \rightarrow Y$ be equivariant rel. $\alpha: A \rightarrow B$; let the. operation of $B$ on $Y$ be simple, and let the orbits in $X$ under $A$ be the fibers of $X \rightarrow T$. Then the orbits in $X$ under $\alpha^{-1}(0)$ are the fibers of $X \rightarrow T \times Y$.

(2.6) Let $A$ operate simply on $X$; let $B$ and $C$ be subgroups of $A$. If the orbits in $X$ under $B$ and $C$ are the fibers of $X \rightarrow S$ and $X \rightarrow T$ respectively, then the orbits under $B \cap C$ are the fibers of $X \rightarrow S \times T$.

(2.7) Let $X \rightarrow^{f} Y \rightarrow^{\circ} C$ be equivariant relative to $A \rightarrow B \rightarrow C$ (where $X$ and $Y$ are sets and $A, B, C$ are groups). If $X$ is nonempty, the operation of $A$ on $X$ is transitive, the operation of $B$ on $Y$ is simple, and $X \rightarrow Y \rightarrow C$ is exact, then so is $A \rightarrow B \rightarrow C$. "Conversely," if $(g f)^{-1}(0)$ is nonempty, the operations of $A$ and $B$ on $X$ and $Y$ respectively are transitive, and $A \rightarrow B \rightarrow C$ is exact, then so is $X \rightarrow Y \rightarrow C$.

(2.8) Let $f: X \rightarrow Y$ be equivariant rel. $\alpha: A \rightarrow B$. If $S$ is an orbit in $X$ under $A$, then $f(S)$ is an orbit in $Y$ under $\alpha(A)$. If $f(X)=Y$, then each orbit in $Y$ under $\alpha(A)$ is the image of some orbit in $X$ under $A$; hence in this case, "the images of the orbits are the orbits under the image."

(2.9) Let (2.3) and (2.4) be diagrams of set-theoretic mappings and homomorphisms respectively. Let $B, C, D$ operate on $Y, Z, W$ respectively, so that 
$Y \rightarrow W$ is equivariant rel. $B \rightarrow D$, and $Z \rightarrow W$ is equivariant rel. $C \rightarrow D$. If (2.3) is strictly regular and (2.4) is commutative, then there exists one and only one operation of $A$ on $X$ such that (2.3) is equivariant rel. (2.4).

(2.10) Let (2.3) be equivariant rel. (2.4). If the operations of $B$ and $C$ on $Y$ and $Z$ respectively are simple, and (2.4) is strict, then the operation of $A$ on $X$ is simple.

(2.11) Let (2.3) be equivariant rel. (2.4). Let the orbits in $Y$ under $B$ be the fibers of $Y \rightarrow S$; let the orbits in $Z$ under $C$ be the fibers of $Z \rightarrow T$. If (2.3) is strict and commutative, and (2.4) is regular, then the orbits in $X$ under $A$ are the fibers of $X \rightarrow S \times T$.

(2.12) Let the set-theoretic map $f: X \rightarrow Y$ and the homomorphism $\alpha: A \rightarrow B$ be onto. Let $A$ operate on $X$ so that the orbits in $X$ under $\alpha^{-1}(0)$ are the fibers of $X \rightarrow Y$. Then there exists one and only one operation of $B$ on $Y$ so that $f$ is equivariant rel. $\alpha$. If the operation of $A$ on $X$ is simple, then so is the operation of $B$ on $Y$. If $g: Y \rightarrow T$ is a mapping such that the orbits in $X$ under $A$ are the fibers of $g f: X \rightarrow T$, then the orbits in $Y$ under $B$ are the fibers of $g: Y \rightarrow T$.

(2.13) Let $X \rightarrow Y$ be equivariant rel. $A \rightarrow B$. Let the mapping $Y \rightarrow Z$ and the homomorphism $B \rightarrow C$ be such that $X \rightarrow Z$ is equivariant rel. $A \rightarrow C$. If $X \rightarrow Y$ and $A \rightarrow B$ are onto, then $Y \rightarrow Z$ is equivariant rel. $B \rightarrow C$.

(2.14) Let (2.3) be regular, and equivariant rel. (2.4); let $D$ be abelian, and let the operation of $D$ on $W$ be simply transitive. Define $\chi: Y \times Z \rightarrow D$ by $\chi(y, z)=h(y)-k(z)$. Define $\rho: B+C \rightarrow D$ by $\rho(b, c)=\gamma(b)-\delta(c)$. Let $B+C$ operate on $Y \times Z$ by $(b, c)+(y, z)=(b+y, c+z)$. Then

$$
X \stackrel{f \times g}{\longrightarrow} Y \times Z \stackrel{\chi}{\longrightarrow} D
$$

is exact, and equivariant relative to

$$
A \stackrel{\alpha \times \beta}{\longrightarrow} B+C \stackrel{\rho}{\longrightarrow} D .
$$

(2.15) Let (2.3) be equivariant relative to (2.4), and let (2.4) be regular. If $X$ is nonempty, the operations of $B$ and $C$ on $Y$ and $Z$ respectively are transitive, the operation of $D$ on $W$ is simple, and (2.3) is commutative, then (2.3) is regular.

3. The sets $\operatorname{CON}, \operatorname{EXT}(\tau), \operatorname{EXT}\left(\phi_{0} \tau\right), \mathrm{P}$. It is suggested that the reader consult diagram (5.4) while reading this section.

Throughout the paper we assume given two fixed groups $Q$ and $K$, a normal subgroup $X$ of $K$, and a homomorphism $\tau: Q \rightarrow A_{X}(K) / I(K)$. We denote $K / X$ by $N$, and the natural homomorphism by $\phi: K \rightarrow N$. We shall use all the symbols introduced on pp. 110-111 of [CGE I], not involving $G$ or $\mu$.

A continuation is a group extension (1.2) of $Q$ by $N$, together with a group $E$ containing $K$ as a subgroup, and a homomorphism $\phi: E \rightarrow G$ satisfying

$$
\bar{\phi} \mid K=\phi, \quad \bar{\phi}(E)=G, \quad \bar{\phi}^{-1}(0)=X, \quad \tau \mu \bar{\phi}=\nu C_{K} .
$$

The last condition means that 


$$
0 \rightarrow K \rightarrow E \stackrel{\mu \Phi}{\rightarrow} Q \rightarrow 0
$$

is an extension of the $Q$-kernel $\tau: Q \rightarrow A_{X}(K) / I(K)$. It follows that (1.2) is an extension of the $Q$-kernel $\phi_{0} \tau: Q \rightarrow A(N) / I(N)$.

Two continuations $(G, \mu, E, \phi)$ and $\left(G^{\prime}, \mu^{\prime}, E^{\prime}, \Phi^{\prime}\right)$ will be called isomorphic if $(G, \mu)=\left(G^{\prime}, \mu^{\prime}\right)$ and there exists a homomorphism $W: E \rightarrow E^{\prime}$ satisfying

$$
W \mid K=\text { identity, } \quad \Phi^{\prime} W=\bar{\phi} .
$$

By the "five-lemma," such a homomorphism is automatically an isomorphism of $E$ onto $E^{\prime}$.

Two continuations $(G, \mu, E, \phi)$ and $\left(G^{\prime}, \mu^{\prime}, E^{\prime}, \Phi^{\prime}\right)$ will be called similar if $(G, \mu)=\left(G^{\prime}, \mu^{\prime}\right)$ and there exist $n_{0} \in Z_{N}$ and a homomorphism $W: E \rightarrow E^{\prime}$ satisfying

$$
W \mid K=\text { identity, } \quad n_{0}+\bar{\phi} e=\bar{\phi}^{\prime} W e+n_{0}
$$

for all $e \in E$. Such a $W$ is automatically an isomorphism of $E$ onto $E^{\prime}$. Condition $(3.4 \mathrm{~b})$ may be written $\bar{\phi}^{\prime} W=\left(c_{G} n_{0}\right) \bar{\phi}$.

CON, CON, and $\mathfrak{S} \subseteq \mathfrak{N}$ will denote, respectively, the set of all continuations, the set of all isomorphism-classes of continuations, and the set of all similarity-classes of continuations. Since any two isomorphic continuations are similar, we have natural mappings $\mathrm{CON} \rightarrow \mathrm{CON} \rightarrow \subseteq \subseteq \mathfrak{N}$.

$\operatorname{EXT}(\tau)$ and $\operatorname{EXT}(\tau)$ will denote, respectively, the set of all extensions of the $Q$-kernel $\tau: Q \rightarrow A_{X}(K) / I(K)$ and the set of all isomorphism-classes of such. $\operatorname{EXT}\left(\phi_{0} \tau\right)$ and $\operatorname{EXT}\left(\phi_{0} \tau\right)$ will denote, respectively, the set of all extensions of the $Q$-kernel $\phi_{0} \tau: Q \rightarrow A(N) / I(N)$ and the set of all isomorphismclasses of such. The passage from $(G, \mu, E, \bar{\phi})$ to $(E, \mu \bar{\phi})$ is a mapping CON $\rightarrow \operatorname{EXT}(\tau)$, which induces a mapping $(\mathfrak{S} \mathfrak{N} \rightarrow \operatorname{EXT}(\tau)$.

A modular expansion is a group extension (1.2) of $Q$ by $N$, together with a modular structure $\theta: G \rightarrow A_{X}(K) / c(X)$ on the normal homomorphism $\phi: K \rightarrow G$ satisfying (1.5). $\mathrm{P}$ will denote the set of all modular expansions. The passage from $(G, \mu, \theta)$ to $(G, \mu)$ is a mapping $\mathrm{P} \rightarrow \operatorname{EXT}\left(\phi_{0} \tau\right)$.

Two modular expansions $(G, \mu, \theta)$ and $\left(G^{\prime}, \mu^{\prime}, \theta^{\prime}\right)$ will be called isomorphic if there exists a homomorphism $W: G \rightarrow G^{\prime}$ satisfying

$$
W \mid N=\text { identity, } \quad \mu^{\prime} W=\mu, \quad \theta^{\prime} W=\theta .
$$

Such a $W$ is automatically an isomorphism of $G$ onto $G^{\prime}$. Two modular expansions $(G, \mu, \theta)$ and $\left(G^{\prime}, \mu^{\prime}, \theta^{\prime}\right)$ will be called congruent if they are isomorphic and $(G, \mu)=\left(G^{\prime}, \mu^{\prime}\right)$. $\mathfrak{B}$ and $\mathbf{P}$ will denote, respectively, the set of all congruence classes of modular expansions and the set of all isomorphism-classes of modular expansions. We have natural mappings $\mathrm{P} \rightarrow \mathfrak{P} \rightarrow \mathbf{P}$. Furthermore, $\mathrm{P} \rightarrow \operatorname{EXT}\left(\phi_{0} \tau\right)$ induces mappings $\mathfrak{B} \rightarrow \operatorname{EXT}\left(\phi_{0} \tau\right)$ and $\mathrm{P} \rightarrow \operatorname{EXT}\left(\phi_{0} \tau\right)$.

Given a continuation $(G, \mu, E, \bar{\phi})$, define $\theta: G \rightarrow A_{X}(K) / c(X)$ by $\theta \bar{\phi}=\lambda C_{K}$ (cf. [CGE I], §3). Then the passage from $(G, \mu, E, \bar{\phi})$ to $(G, \mu, \theta)$ is a mapping $\mathrm{CON} \rightarrow \mathrm{P}$, which induces mappings $\mathrm{CON} \rightarrow \mathrm{P}$ and $\mathfrak{S} S \mathfrak{N} \rightarrow \mathfrak{B}$. 
Given an extension

$$
0 \rightarrow K \rightarrow E \stackrel{\rho}{\rightarrow} Q \rightarrow 0
$$

of the $Q$-kernel $\tau: Q \rightarrow A_{X}(K) / I(K)$, let $G=E / X$, and let $\phi: E \rightarrow G$ denote the natural homomorphism. Then $\rho: E \rightarrow Q$ induces a homomorphism $\mu: G \rightarrow Q$, defined by $\mu \bar{\phi}=\rho$; and $(G, \mu, E, \phi)$ is a continuation. This construction defines a mapping $\operatorname{EXT}(\tau) \rightarrow \mathrm{CON}$, which is a right inverse of $\mathrm{CON} \rightarrow \operatorname{EXT}(\tau)$, showing that the latter mapping and hence also $\mathfrak{S} \subseteq \mathfrak{N} \rightarrow \operatorname{EXT}(\tau)$ are onto. Since $\operatorname{EXT}(\tau) \rightarrow \operatorname{CON}$ does not commute with all the mappings defined previously, it will, for the most part, be ignored. However, the mapping $\operatorname{EXT}(\tau)$ $\rightarrow \mathbf{P}$ obtained from the composition $\operatorname{EXT}(\tau) \rightarrow \mathrm{CON} \rightarrow \mathbf{P} \rightarrow \mathfrak{P} \rightarrow \mathbf{P}$ does commute with the other mappings, and will be included in our investigation. $\operatorname{EXT}(\tau) \rightarrow \mathbf{P}$ induces a mapping $\operatorname{EXT}(\tau) \rightarrow \mathbf{P}$.

If $(G, \mu) \in \operatorname{EXT}\left(\phi_{0} \tau\right)$, and $T=\mathrm{CON}, \mathrm{CON}$, $\subseteq \subseteq \mathfrak{N}, \mathrm{P}$, or $\mathfrak{B}$, then $T_{(G, \mu)}$ will denote the counter-image of $(G, \mu)$ under the mapping $T \rightarrow \operatorname{EXT}\left(\phi_{0} \tau\right)$.

4. Cohomology groups. The following symbols will denote the indicated natural homomorphisms:

$$
i: X \cap Z_{K} \rightarrow Z_{K} ; \quad j: \phi Z_{K} \rightarrow Z_{N} ; \quad \alpha: N \rightarrow N / \phi Z_{K} .
$$

The maps $Z_{K} \rightarrow \phi Z_{K}$ and $Z_{N} \rightarrow Z_{N} / \phi Z_{K}$ obtained by restricting $\phi$ and $\alpha$ respectively will be denoted by the same symbols $\phi$ and $\alpha$.

Given any cochain complex $C, \rho: Z^{n} \rightarrow H^{n}$ will denote the natural mapping of the group of $n$-dimensional cocycles onto the $n$-dimensional cohomology group.

$\alpha: N \rightarrow N / \phi Z_{K}$ induces homomorphisms

$$
\alpha_{f}: A_{\phi Z_{K}}(N) \rightarrow A\left(N / \phi Z_{K}\right), \quad \alpha_{0}: A_{\phi Z_{K}}(N) / I(N) \rightarrow A\left(N / \phi Z_{K}\right) / I\left(N / \phi Z_{K}\right)
$$

analogous to those induced by $\phi: K \rightarrow N$ [CGE I, p. 110]. Clearly $\phi_{0} \tau(Q)$ $\subset A_{\phi Z_{K}}(N) / I(N)$.

If $Y=X \cap Z_{K}, Z_{K}, \phi Z_{K}, Z_{N}, Z_{N} / \phi Z_{K}, Z_{\alpha N}$, or $Z_{\alpha N} / \alpha Z_{N}$, the cohomology groups $H^{n}(Q, Y)$ are to be understood as defined in terms of the operation of $Q$ and $Y$ induced by $\tau: Q \rightarrow A_{X}(K) / I(K), \phi_{0} \tau: Q \rightarrow A(N) / I(N)$, or $\alpha_{0} \phi_{0} \tau: Q$ $\rightarrow A(\alpha N) / I(\alpha N)$.

Let $C^{n}(\phi)$ denote the direct sum $C^{n}\left(Q, Z_{K}\right)+C^{n-1}\left(Q, Z_{N}\right)$. In particular, $C^{0}(\phi)=Z_{K}$, and $C^{n}(\phi)=0$ for $n<0$. Define $D: C^{n}(\phi) \rightarrow C^{n+1}(\phi)$ by

$$
D(f, g)=\left[\delta f, \delta g+(-1)^{n-1} \phi f\right]
$$

(cf. [E-S, p. 155]). Then $D D=0$. Let

$$
Z^{n}(\phi)=C^{n}(\phi) \cap D^{-1}(0), B^{n}(\phi)=D\left[C^{n-1}(\phi)\right], H^{n}(\phi)=Z^{n}(\phi) / B^{n}(\phi) .
$$

Define 


$$
\begin{aligned}
\epsilon_{K}: C^{n}\left(Q, Z_{K}\right) & \rightarrow C^{n}(\phi), & \epsilon_{N}: C^{n-1}\left(Q, Z_{N}\right) & \rightarrow C^{n}(\phi), \\
\pi_{K}: C^{n}(\phi) & \rightarrow C^{n}\left(Q, Z_{K}\right), & \pi_{N}: C^{n}(\phi) & \rightarrow C^{n-1}\left(Q ; Z_{N}\right)
\end{aligned}
$$

by

$$
\epsilon_{K}(f)=(f, 0), \quad \epsilon_{N}(g)=(0, g), \quad \pi_{K}(f, g)=f, \quad \pi_{N}(f, g)=g .
$$

Then the homomorphisms

$$
\begin{array}{rlrl}
\epsilon_{K} i: C^{n}\left(Q, X \cap Z_{K}\right) & \rightarrow C^{n}(\phi), & \epsilon_{N}: C^{n-1}\left(Q, Z_{N}\right) & \rightarrow C^{n}(\phi), \\
\pi_{K}: C^{n}(\phi) & \rightarrow C^{n}\left(Q, Z_{K}\right), \quad \alpha \pi_{N}: C^{n}(\phi) \rightarrow C^{n-1}\left(Q, Z_{N} / \phi Z_{K}\right)
\end{array}
$$

are cochain mappings. We obtain a diagram
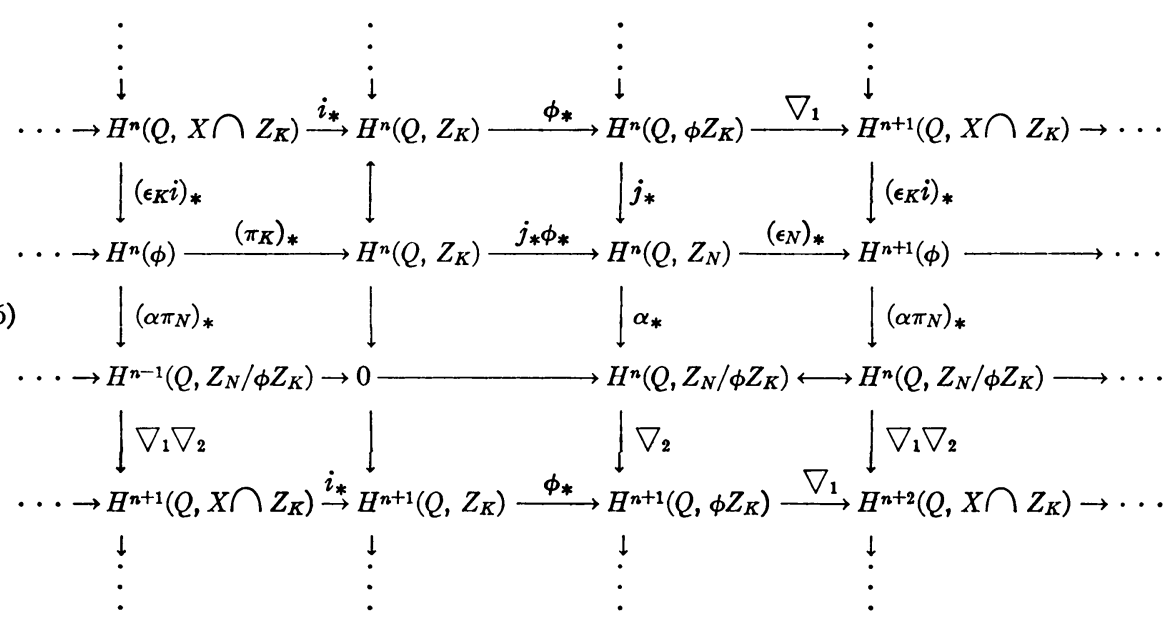

in which all rows and columns are exact, and each small rectangle is commutative, except for the one in the upper right-hand corner, which is anticommutative for $n$ odd: that is, for $n$ odd, the sum of the maps $\left(\epsilon_{K} i\right)_{*} \nabla_{1}$ : $H^{n}\left(Q, \phi Z_{K}\right) \rightarrow H^{n+1}(\phi)$ and $\left(\epsilon_{N}\right)_{*} j_{*}: H^{n}\left(Q, \phi Z_{K}\right) \rightarrow H^{n+1}(\phi)$ is zero.

The presence of 0 in (4.6) gives rise to an "involution" of the diagram, in which the same nontrivial exact sequences occur in a different arrangement. Each small rectangle in the involuted diagram is commutative, except for

$$
\begin{array}{ll}
H^{n}(\phi) \stackrel{\left(\pi_{K}\right)_{*}}{\longrightarrow} H^{n}\left(Q, Z_{K}\right) \\
\downarrow\left(\alpha \pi_{N}\right)_{*} \\
H^{n-1}\left(Q, Z_{N} / \phi Z_{K}\right) \stackrel{\nabla_{2}}{\longrightarrow} H^{n}\left(Q, \phi Z_{K}\right),
\end{array}
$$

which is anti-commutative for $n$ odd. 
It follows from these assertions that the sequences

$$
\begin{aligned}
\cdots \rightarrow & H^{n}\left(Q, X \cap Z_{K}\right)+H^{n-1}\left(Q, Z_{N}\right) \stackrel{\left(\epsilon_{K} i\right)_{*}+(-1)^{n}\left(\epsilon_{N}\right)_{*}}{\longrightarrow} H^{n}(\phi) \\
& \stackrel{\phi_{*}\left(\pi_{K}\right)_{*}}{\longrightarrow} H^{n}\left(Q, \phi Z_{K}\right) \stackrel{\nabla_{1}+j_{*}}{\longrightarrow} H^{n+1}\left(Q, X \cap Z_{K}\right)+H^{n}\left(Q, Z_{N}\right) \rightarrow \cdots \\
\cdots \rightarrow & H^{n}\left(Q, Z_{K}\right)+H^{n-1}\left(Q, Z_{N} / \phi Z_{K}\right) \stackrel{\phi_{*}+(-1)^{n-1} \nabla_{2}}{\longrightarrow} H^{n}\left(Q, \phi Z_{K}\right) \\
& \stackrel{\left(\epsilon_{N}\right)_{*} j_{*}}{\longrightarrow} H^{n+1}(\phi) \stackrel{\left(\pi_{K}\right)_{*}+\left(\alpha \pi_{N}\right)_{*}}{\longrightarrow} H^{n+1}\left(Q, Z_{K}\right)+H^{n}\left(Q, Z_{N} / \phi Z_{K}\right) \rightarrow \cdots
\end{aligned}
$$

are exact. They remain exact if $\phi_{*}\left(\pi_{K}\right)_{*}$ is replaced by $\nabla_{\mathbf{2}}\left(\alpha \pi_{N}\right)_{*}$, and/or $\left(\epsilon_{N}\right)_{*} j_{*}$ is replaced by $\left(\epsilon_{K} i\right)_{*} \nabla_{1}$.

Let $\widetilde{B}^{n}(\phi)=D \epsilon_{K}\left[C^{n-1}\left(Q, Z_{K}\right)\right], \widetilde{H}^{n}(\phi)=Z^{n}(\phi) / \widetilde{B}^{n}(\phi)$. Let $\beta: \widetilde{H}^{n}(\phi) \rightarrow H^{n}(\phi)$ denote the natural homomorphism. $\epsilon_{K} i: C^{n}\left(Q, \quad X \cap Z_{K}\right) \rightarrow C^{n}(\phi)$ maps $B^{n}\left(Q, X \cap Z_{K}\right)$ into $\widetilde{B}^{n}(\phi)$, and therefore induces a homomorphism $\left(\epsilon_{K} i\right)_{\sharp}$ : $H^{n}\left(Q, X \cap Z_{K}\right) \rightarrow \widetilde{H}^{n}(\phi) . \alpha \pi_{N}: C^{n}(\phi) \rightarrow C^{n-1}\left(Q, Z_{N} / \phi Z_{K}\right)$ maps $\widetilde{B}^{n}(\phi)$ to zero, and therefore induces a homomorphism $\left(\alpha \pi_{N}\right): \widetilde{H}^{n}(\phi) \rightarrow Z^{n-1}\left(Q, Z_{N} / \phi Z_{K}\right)$. $\epsilon_{N}: C^{n-1}\left(Q, Z_{N}\right) \rightarrow C^{n}(\phi)$ carries the subgroup $\phi Z^{n-1}\left(Q, Z_{K}\right)$ of $C^{n-1}\left(Q, Z_{N}\right)$ into $\widetilde{B}^{n}(\phi)$, and therefore induces a homomorphism

$$
\left(\epsilon_{N}\right) \sharp Z^{n-1}\left(Q, Z_{N}\right) / \phi Z^{n-1}\left(Q, Z_{K}\right) \rightarrow \widetilde{H}^{n}(\phi) .
$$

Commutativity holds throughout the diagram

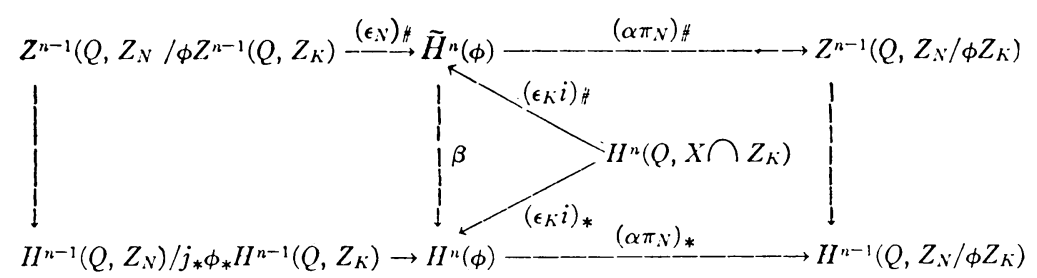

where the unlabelled horizontal homomorphism is induced by $\left(\epsilon_{N}\right)_{*}$ : $H^{n-1}\left(Q, Z_{N}\right) \rightarrow H^{n}(\phi)$. For each $n$, the sequences

$$
\begin{aligned}
0 \rightarrow Z^{n-1}\left(Q, Z_{N}\right) / \phi Z^{n-1}\left(Q, Z_{K}\right) & \stackrel{\left(\epsilon_{N}\right)_{\star}}{\longrightarrow} \tilde{H}^{n}(\phi) \stackrel{\left(\pi_{K}\right)_{*} \beta}{\longrightarrow} H^{n}\left(Q, Z_{K}\right) \\
& \stackrel{j_{*} \phi_{*}}{\longrightarrow} H^{n}\left(Q, Z_{N}\right) \stackrel{\left(\epsilon_{N}\right)_{*}}{\longrightarrow} H^{n+1}(\phi) \rightarrow \cdots
\end{aligned}
$$

$$
\begin{aligned}
0 \rightarrow H^{n}\left(Q, X \cap Z_{K}\right) \stackrel{\left(\epsilon_{K} i\right)_{\boldsymbol{t}}}{\longrightarrow} \tilde{H}^{n}(\phi) \stackrel{\left(\alpha \pi_{N}\right)_{\boldsymbol{H}}}{\longrightarrow} Z^{n-1}\left(Q, Z_{N} / \phi Z_{K}\right) \\
\quad \stackrel{\nabla_{1} \nabla_{2} \rho}{\longrightarrow} H^{n+1}\left(Q, X \cap Z_{K}\right) \stackrel{\left(\epsilon_{K} i\right)_{*}}{\longrightarrow} H^{n+1}(\phi) \rightarrow \cdots
\end{aligned}
$$

are exact. With the finite number of exceptions shown, (4.11) and (4.12) coincide with sequences from (4.6). 
All of the above holds, of course, if the exact sequence

$$
0 \rightarrow C^{n}\left(Q, X \cap Z_{K}\right) \rightarrow C^{n}\left(Q, Z_{K}\right) \stackrel{j \phi}{\rightarrow} C^{n}\left(Q, Z_{N}\right) \rightarrow C^{n}\left(Q, Z_{N} / \phi Z_{K}\right) \rightarrow 0
$$

is replaced by an arbitrary exact sequence (of the same length) of cochain complexes.

5. The main results. We recall from [CTAG II], and Theorem 5.12 of [CGE I], that $H^{2}\left(Q, Z_{K}\right)$ and $H^{2}\left(Q, Z_{N}\right)$ operate in a natural way as simply transitive transformation groups on $\operatorname{EXT}(\tau)$ and $\operatorname{EXT}\left(\phi_{0} \tau\right)$ respectively. By Theorem 10.2 of [CGE I], $H^{2}\left(Q, X \cap Z_{K}\right)$ operates naturally as a simply transitive transformation group on each fiber of $\mathrm{CON} \rightarrow \mathrm{P}$; hence we may say that $H^{2}\left(Q, X \cap Z_{K}\right)$ operates simply on CON, the orbits being the fibers of $\mathrm{CON} \rightarrow \mathrm{P}$. The notion of the "obstruction of a pseudo-module," defined in [CGE I], provides a natural mapping $\chi: \mathrm{P} \rightarrow H^{3}\left(Q, X \cap Z_{K}\right)$. It is easily verified that any two isomorphic modular expansions have the same obstruction; hence $\chi: \mathrm{P} \rightarrow H^{3}\left(Q, X \cap Z_{K}\right)$ induces a mapping $\chi: \mathrm{P} \rightarrow H^{3}\left(Q, X \cap Z_{K}\right)$. By Corollary 10.3 of [CGE I], the sequence

$$
\mathrm{CON} \rightarrow \mathrm{P} \stackrel{\chi}{\rightarrow} H^{3}\left(Q, X \cap Z_{K}\right)
$$

is exact; hence so is

$$
\operatorname{EXT}(\tau) \rightarrow \mathrm{P} \stackrel{\chi}{\rightarrow} H^{3}\left(Q, X \cap Z_{K}\right) .
$$

THeOREM 5.3. There exist a natural mapping $\chi: \operatorname{EXT}\left(\phi_{0} \tau\right) \rightarrow H^{3}(\phi)$, natural operations of $Z^{1}\left(Q, \phi Z_{K}\right)$ and $Z^{1}\left(Q, Z_{N}\right)$ on $C O N$, and natural operations of $\tilde{H}^{2}(\phi), H^{2}(\phi), Z^{1}\left(Q, Z_{N} / \phi Z_{K}\right), \nabla_{2} H^{1}\left(Q, Z_{N} / \phi Z_{K}\right), H^{2}\left(Q, \phi Z_{K}\right)$ on CON, $\mathfrak{S} \bigcirc \mathfrak{N}, \mathrm{P}, \mathfrak{B}, \mathbf{P}$ respectively, such that the diagram

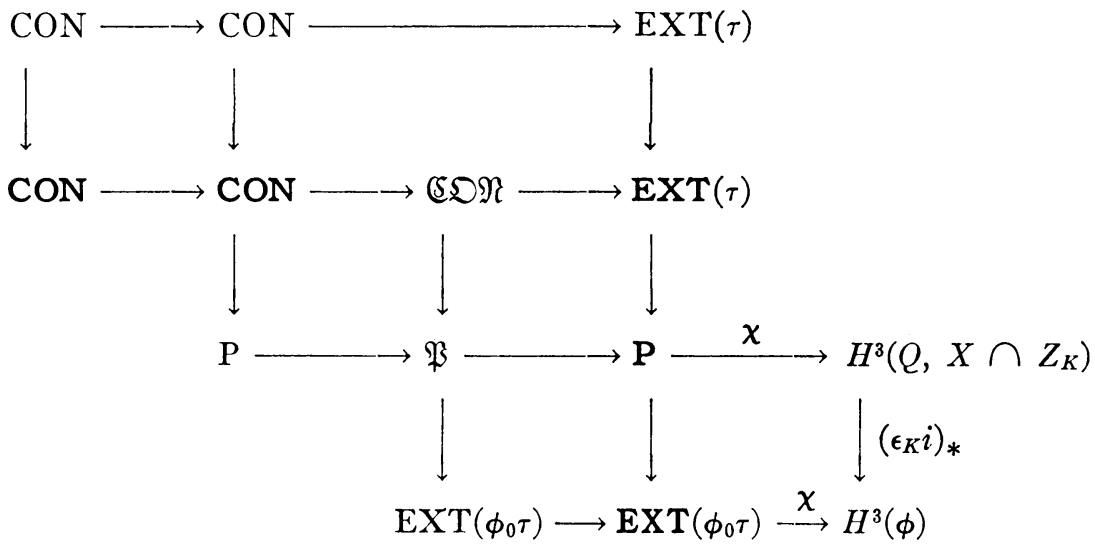

is equivariant relative to the diagram 

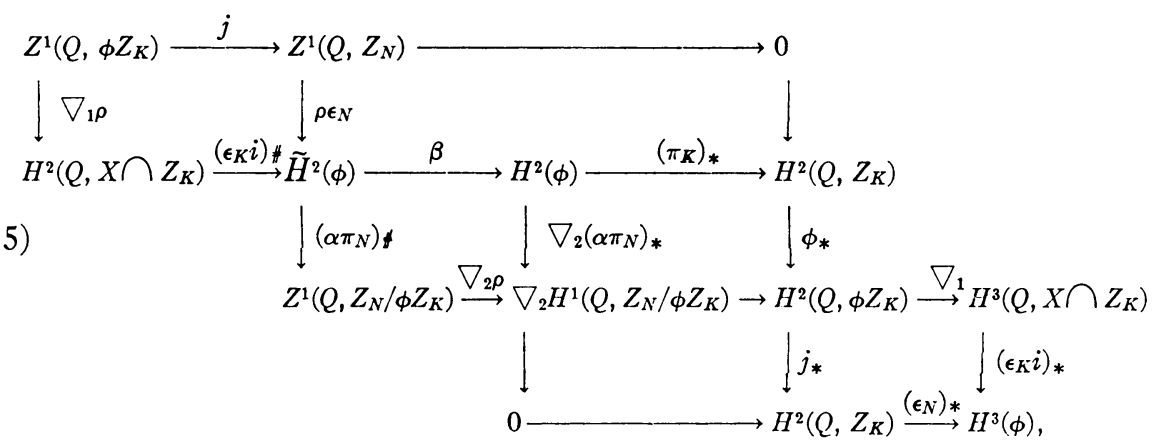

except that $\mathrm{CON} \rightarrow \mathrm{CON}$ is antivariant rel. $\nabla_{1} \rho: Z^{1}\left(Q, \phi Z_{K}\right) \rightarrow H^{2}\left(Q, X \cap Z_{K}\right)$. All the operations are simple. The operation of $H^{2}\left(Q, \phi Z_{K}\right)$ on $\mathbf{P}$ is simply transitive. The orbits in $\mathrm{CON}$ under $Z^{1}\left(Q, \phi Z_{K}\right)$ and $Z^{1}\left(Q, Z_{N}\right)$ are the fibers of $\mathrm{CON}$ $\rightarrow \mathrm{P} \times \operatorname{EXT}(\tau)$ and $\mathrm{CON} \rightarrow \operatorname{EXT}\left(\phi_{0} \tau\right) \times \operatorname{EXT}(\tau)$ respectively. The orbits in $\mathrm{CON}$ under $\widetilde{H}^{2}(\phi)$ are the fibers of $\mathrm{CON} \rightarrow \mathrm{EXT}\left(\phi_{0} \tau\right)$. If $S=(5 \subseteq \mathfrak{N}, \mathrm{P}$, or $\mathfrak{B}$, then the orbits in $S$ under the corresponding group are the fibers of $S \rightarrow \operatorname{EXT}\left(\phi_{0} \tau\right)$.

We note that diagrams (5.4) and (5.5) are commutative, except for the upper left-hand rectangle of (5.5), which is anti-commutative.

THEOREM 5.6. In diagram (5.4), the rectangles from $\mathbb{S O N}$ to $\mathbf{P}$ and from $\mathbf{P}$ to $H^{3}(\phi)$ are regular. The rectangle from $\mathfrak{B}$ to $\operatorname{EXT}\left(\phi_{0} \tau\right)$ is strictly regular. The rectangles

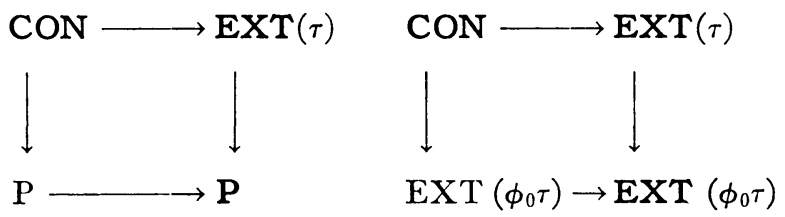

are regular.

The assertions concerning (5.7a) and the rectangle from $\mathfrak{B}$ to $\operatorname{EXT}\left(\phi_{0} \tau\right)$ may be verified directly from the definitions in $\S 3$. Since $\mathrm{P} \rightarrow \mathfrak{P}$ is onto, the regularity of the rectangle from $\mathbb{S} \subseteq \mathfrak{N}$ to $\mathbf{P}$, and hence of $(5.7 \mathrm{~b})$, follows automatically. Consequently the only part of Theorem 5.6 which we shall prove in the sequel is the regularity of the rectangle from $\mathbf{P}$ to $H^{3}(\phi)$.

THEOREM 5.8. The sequences

$$
\begin{gathered}
\operatorname{EXT}(\tau) \rightarrow \operatorname{EXT}\left(\phi_{0} \tau\right) \stackrel{\chi}{\rightarrow} H^{3}(\phi) \\
\mathbf{P} \rightarrow \operatorname{EXT}\left(\phi_{0} \tau\right) \stackrel{\left(\alpha \pi_{N}\right)_{*} \chi}{\longrightarrow} H^{2}\left(Q, Z_{N} / \phi Z_{K}\right)
\end{gathered}
$$

are exact. 
The fact that all the unlabelled horizontal maps in (5.4) are onto yields many equivalent formulations of Theorems 5.6 and 5.8. In particular, the exactness of (5.9) is equivalent to the exactness of (1.4).

$\chi(\tau) \in H^{3}\left(Q, Z_{K}\right)$ and $\chi\left(\phi_{0} \tau\right) \in H^{3}\left(Q, Z_{N}\right)$ will denote, respectively, the obstructions [CTAG II] of the $Q$-kernels $\tau: Q \rightarrow A_{X}(K) / I(K)$ and $\phi_{0} \tau: Q$ $\rightarrow A(N) / I(N)$.

Theorem 5.11. The subset $\chi(\mathbf{P})$ of $H^{3}\left(Q, X \cap Z_{K}\right)$ is equal to $i_{*}^{-1}[\chi(\tau)]$. The subset $\chi\left[\operatorname{EXT}\left(\phi_{0} \tau\right)\right]$ of $H^{3}(\phi)$ is equal to $\left(\pi_{K}\right)_{*}^{-1}[\chi(\tau)]$.

Theorem 5.11 holds whether the sets in question are empty or not. Thus the "empty" case of Theorem 5.11a asserts that there exists a modular expansion if and only if $\chi(\tau) \in i_{*} H^{3}\left(Q, X \cap Z_{K}\right)$, i.e. if and only if the element $\phi_{*} \chi(\tau)$ of $H^{3}\left(Q, \phi Z_{K}\right)$ vanishes (cf. [APC, Theorem 1]). The "empty" case of Theorem $5.11 \mathrm{~b}$ asserts that the $Q$-kernel $\phi_{0} \tau: Q \rightarrow A(N) / I(N)$ is extendible if and only if $\chi(\tau) \in\left(\pi_{K}\right)_{*} H^{3}(\phi)$, i.e. if and only if $j_{*} \phi_{*} \chi(\tau)=0$. Actually, it is easily verified that

$$
j_{*} \phi_{*} \chi(\tau)=\chi\left(\phi_{0} \tau\right)
$$

(cf. [APC]). Hence, in the sequel, we shall prove $5.11 \mathrm{~b}$ only in the nonempty case.

6. Corollaries. Applying (2.14) to Theorem 5.6, we find that

$$
\begin{aligned}
& \left(\mathfrak{S N} \rightarrow \mathfrak{B} \times \operatorname{EXT}(\tau) \rightarrow H^{2}\left(Q, \phi Z_{K}\right),\right. \\
& \mathrm{P} \rightarrow \operatorname{EXT}\left(\phi_{0} \tau\right) \times H^{3}\left(Q, X \cap Z_{K}\right) \rightarrow H^{3}(\phi), \\
& \overline{\mathfrak{B}} \rightarrow \operatorname{EXT}\left(\phi_{0} \tau\right) \times \mathrm{P} \rightarrow H^{2}\left(Q, Z_{N}\right), \\
& \operatorname{CON} \rightarrow \mathrm{P} \times \operatorname{EXT}(\tau) \rightarrow H^{2}\left(Q, \phi Z_{K}\right), \\
& \operatorname{CON} \rightarrow \operatorname{EXT}\left(\phi_{0} \tau\right) \times \operatorname{EXT}(\tau) \rightarrow H^{2}\left(Q, Z_{N}\right)
\end{aligned}
$$

form exact sequences, equivariant relative to

$$
\begin{aligned}
& H^{2}(\phi) \rightarrow \nabla_{2} H^{1}\left(Q, Z_{N} / \phi Z_{K}\right)+H^{2}\left(Q, Z_{K}\right) \rightarrow H^{2}\left(Q, \phi Z_{K}\right), \\
& H^{2}\left(Q, \phi Z_{K}\right) \rightarrow H^{2}\left(Q, Z_{N}\right)+H^{3}\left(Q, X \cap Z_{K}\right) \rightarrow H^{3}(\phi), \\
& \nabla_{2} H^{1}\left(Q, Z_{N} / \phi Z_{K}\right) \rightarrow H^{2}\left(Q, \phi Z_{K}\right) \rightarrow H^{2}\left(Q, Z_{N}\right), \\
& \tilde{H}^{2}(\phi) \rightarrow Z^{1}\left(Q, Z_{N} / \phi Z_{K}\right)+H^{2}\left(Q, Z_{K}\right) \rightarrow H^{2}\left(Q, \phi Z_{K}\right), \\
& \widetilde{H}^{2}(\phi) \rightarrow H^{2}\left(Q, Z_{K}\right) \rightarrow H^{2}\left(Q, Z_{N}\right)
\end{aligned}
$$

respectively. The exact sequence (6.1) [equivalently, (6.4)] yields, in a sense, a cohomological criterion for the existence of a continuation which determines simultaneously a given modular expansion and a given extension of the $Q$-kernel $\tau: Q \rightarrow A_{X}(K) / I(X)$. The other sequences may be interpreted similarly.

Applying (2.5) and (2.6) to Theorem 5.3, we find that: 


\begin{tabular}{|c|c|c|c|}
\hline $\begin{array}{c}\text { The } \\
\text { orbits in }\end{array}$ & under the subgroup & of & are the fibers of \\
\hline $\mathrm{CON}$ & $\phi Z^{1}\left(Q, Z_{K}\right)$ & $Z^{1}\left(Q, Z_{N}\right)$ & $\mathrm{CON} \rightarrow \mathrm{CON} \times \operatorname{EXT}(\tau)$ \\
\hline $\mathrm{CON}$ & $Z^{1}\left(Q, \phi Z_{K}\right)$ & $Z^{1}\left(Q, Z_{N}\right)$ & $\mathrm{CON} \rightarrow \mathrm{P} \times \operatorname{EXT}(\tau)$ \\
\hline $\mathrm{CON}$ & $\rho \epsilon_{N} Z^{1}\left(Q, Z_{N}\right)$ & $\widetilde{H}^{2}(\phi)$ & $\mathrm{CON} \rightarrow \operatorname{EXT}\left(\phi_{0} \tau\right) \times \operatorname{EXT}(\tau)$ \\
\hline CON & $\overline{\left(\epsilon_{K} i\right)_{\sharp} \mathrm{H}^{2}\left(Q, X \cap Z_{K}\right)}$ & $\tilde{H}^{2}(\phi)$ & $\mathrm{CON} \rightarrow \mathrm{P}$ \\
\hline CON & {$\left[\phi_{*}\left(\pi_{K}\right) * \beta\right]^{-1}(0)$} & $\tilde{H}^{2}(\phi)$ & $\mathrm{CON} \rightarrow \mathfrak{P}$ \\
\hline CON & $\left(\epsilon_{K} i\right)_{\#} \nabla_{1} H^{1}\left(Q, \phi Z_{K}\right)$ & $\tilde{H}^{2}(\phi)$ & $\mathrm{CON} \rightarrow \operatorname{P} \times \operatorname{EXT}(\tau)$ \\
\hline $\operatorname{EXT}(\tau)$ & $i_{*} H^{2}\left(Q, X \cap Z_{K}\right)$ & $H^{2}\left(Q, Z_{K}\right)$ & $\operatorname{EXT}(\tau) \rightarrow \mathbf{P}$ \\
\hline $\operatorname{EXT}(\tau)$ & $\left(\pi_{K}\right) * H^{2}(\phi)$ & $H^{2}\left(Q, Z_{K}\right)$ & $\operatorname{EXT}(\tau) \rightarrow \operatorname{EXT}\left(\phi_{0} \tau\right)$ \\
\hline $\mathrm{P}$ & $\alpha Z^{1}\left(Q, Z_{N}\right)$ & $Z^{1}\left(Q, Z_{N} / \phi Z_{K}\right)$ & $\mathrm{P} \rightarrow \mathfrak{P}$ \\
\hline $\mathrm{P}$ & $\left(\alpha \pi_{N}\right) \sharp \widetilde{H}^{2}(\phi)$ & $Z^{1}\left(Q, Z_{N} / \phi Z_{K}\right)$ & $\mathrm{P} \rightarrow \operatorname{EXT}\left(\phi_{0} \tau\right) \times H^{3}\left(Q, X \cap Z_{K}\right)$ \\
\hline $\mathfrak{B}$ & $\nabla_{2} H^{1}\left(Q, Z_{N} / \phi Z_{K}\right) \bigcap_{\phi *} H^{2}\left(Q, Z_{K}\right)$ & $\nabla_{2} H^{1}\left(Q, Z_{N} / \phi Z_{K}\right)$ & $\mathfrak{P} \rightarrow \operatorname{EXT}\left(\phi_{0} \tau\right) \times H^{3}\left(Q, X \bigcap Z_{K}\right)$ \\
\hline $\mathbf{P}$ & $\phi_{*} H^{2}\left(Q, Z_{K}\right)$ & $H^{2}\left(Q, \phi Z_{K}\right)$ & $\mathbf{P} \stackrel{x}{\rightarrow} H^{3}\left(Q, X \cap Z_{K}\right)$ \\
\hline $\mathbf{P}$ & $\nabla_{2} H^{1}\left(Q, Z_{N} / \phi Z_{K}\right)$ & $H^{2}\left(Q, \phi Z_{K}\right)$ & $\mathbf{P} \rightarrow \operatorname{EXT}\left(\phi_{0} \tau\right)$ \\
\hline $\mathbf{P}$ & $\nabla_{2} H^{1}\left(Q, Z_{N} / \phi Z_{K}\right) \bigcap_{\phi_{*}} H^{2}\left(Q, Z_{K}\right)$ & $H^{2}\left(Q, \phi Z_{K}\right)$ & $\mathbf{P} \rightarrow \operatorname{EXT}\left(\phi_{0} \tau\right) \times H^{3}\left(Q, X \cap Z_{K}\right)$ \\
\hline $\operatorname{EXT}\left(\phi_{0} \tau\right)$ & $j_{* \phi *} H^{2}\left(Q, Z_{K}\right)$ & $H^{2}\left(Q, Z_{N}\right)$ & $\operatorname{EXT}\left(\phi_{0} \tau\right) \stackrel{\chi}{\rightarrow} H^{3}(\phi)$ \\
\hline $\operatorname{EXT}\left(\phi_{0} \tau\right)$ & $j_{*} H^{2}\left(Q, \phi Z_{K}\right)$ & $H^{2}\left(Q, Z_{N}\right)$ & $\operatorname{EXT}\left(\phi_{0} \tau\right) \stackrel{\left(\alpha \pi_{N}\right) * \chi}{\longrightarrow} H^{2}\left(Q, Z_{N} / \phi Z_{K}\right)$ \\
\hline
\end{tabular}

In the table on the following page we assert that the domain (and hence the image) of the mapping in the second column is nonempty if and only if the condition in the first column is satisfied; and that in the nonempty case, the image of the mapping is an orbit under the subgroup in the third column of the group in the fourth column. $(G, \mu)$ is an arbitrary element of $\operatorname{EXT}\left(\phi_{0} \tau\right)$; $\chi(G, \mu)$ is the image of $(G, \mu)$ under the composition $\operatorname{EXT}\left(\phi_{0} \tau\right) \rightarrow \operatorname{EXT}\left(\phi_{0} \tau\right)$ $\rightarrow x H^{3}(\phi)$.

In the last three rows, of course, "orbit" means "coset." The assertions concerning the images are applications of (2.8). The third row of the table generalizes Theorems 1 and 2 of [APC]. 


\begin{tabular}{|c|c|c|c|}
\hline$\chi(\tau)=0$ & $\operatorname{EXT}(\tau) \rightarrow \mathbf{P}$ & $\phi_{*} H^{2}\left(Q, Z_{K}\right)$ & $H^{2}\left(Q, \phi Z_{K}\right)$ \\
\hline$\chi(\tau)=0$ & $\operatorname{EXT}(\tau) \rightarrow \operatorname{EXT}\left(\phi_{0} \tau\right)$ & $j_{* \phi *} H^{2}\left(Q, Z_{K}\right)$ & $H^{2}\left(Q, Z_{N}\right)$ \\
\hline$\phi * \chi(\tau)=0$ & $\mathbf{P} \rightarrow \operatorname{EXT}\left(\phi_{0} \tau\right)$ & $j_{*} H^{2}\left(Q, \phi Z_{K}\right)$ & $H^{2}\left(Q, Z_{N}\right)$ \\
\hline$\phi * \chi(\tau)=0$ & $\mathbf{P} \rightarrow \operatorname{EXT}\left(\phi_{0} \tau\right) \times H^{3}\left(Q, X \cap Z_{K}\right)$ & $\begin{array}{l}\text { all }(a, b) \text { such that } \\
\left(\epsilon_{N}\right)_{*}(a)=\left(\epsilon_{K} i\right)_{*}(b)\end{array}$ & $H^{2}\left(Q, Z_{N}\right)+H^{3}\left(Q, X \cap Z_{K}\right)$ \\
\hline$\chi(G, \mu)=0$ & $\mathrm{CON}_{(G, \mu)} \rightarrow \mathrm{P}_{(G, \mu)}$ & $\left(\alpha \pi_{N}\right)_{\#} \widetilde{H}^{2}(\phi)$ & $Z^{1}\left(Q, Z_{N} / \phi Z_{K}\right)$ \\
\hline$\chi(G, \mu)=0$ & $\operatorname{CON}_{(G, \mu)} \rightarrow \mathfrak{P}_{(G, \mu)}$ & $\begin{array}{c}\nabla_{2} H^{1}\left(Q, Z_{N} / \phi Z_{K}\right) \\
\bigcap_{\phi *} H^{2}\left(Q, Z_{K}\right)\end{array}$ & $\nabla_{2} H^{1}\left(Q, Z_{N} / \phi Z_{K}\right)$ \\
\hline$\chi(G, \mu)=0$ & $\operatorname{CON}_{(G, \mu)} \rightarrow \mathrm{P}_{(G, \mu)} \times \operatorname{EXT}(\tau)$ & $\begin{array}{l}\text { all }(a, b) \text { such that } \\
\nabla_{2} \rho(a)=\phi_{*}(b)\end{array}$ & $Z^{1}\left(Q, Z_{N} / \phi Z_{K}\right)+H^{2}\left(Q, Z_{K}\right)$ \\
\hline$\chi(G, \mu)=0$ & $\operatorname{CON}_{(G, \mu)} \rightarrow \mathfrak{B}_{(G, \mu)} \times \operatorname{EXT}(\tau)$ & $\begin{array}{l}\text { all }(a, b) \text { such that } \\
a=\phi *(b)\end{array}$ & $\nabla_{2} H^{1}\left(Q, Z_{N} / \phi Z_{K}\right)+H^{2}\left(Q, Z_{K}\right)$ \\
\hline$\phi * \chi(\tau)=0$ & $\mathbf{p} \stackrel{\chi}{\rightarrow}^{3}\left(\ddot{H} Q, X \cap Z_{K}\right)$ & $\nabla_{1} H^{2}\left(Q, \phi Z_{K}\right)$ & $H^{3}\left(Q, X \bigcap Z_{K}\right)$ \\
\hline$\phi * \chi(\tau)=0$ & $\mathbf{P} \stackrel{\left(\epsilon_{K} i\right) * \chi}{\longrightarrow} H^{3}(\phi)$ & $\left(\epsilon_{K} i\right) * \nabla_{1} H^{2}\left(Q, \phi Z_{K}\right)$ & $H^{3}(\phi)$ \\
\hline $\begin{array}{r}\left(\alpha \pi_{N}\right) * \chi(G, \mu) \\
=0\end{array}$ & $\mathrm{P}_{(\boldsymbol{\theta}, \mu)} \rightarrow H^{3}\left(Q, X \bigcap Z_{K}\right)$ & $\nabla_{1} \nabla_{2} H^{1}\left(Q, Z_{N} / \phi Z_{K}\right)$ & $H^{3}\left(Q, X \bigcap Z_{K}\right)$ \\
\hline
\end{tabular}

PART II

7. The role of $H^{n}(\phi)$ and $\tilde{H}^{2}(\phi)$. In this section we shall define the operations of $\widetilde{H}^{2}(\phi)$ and $H^{2}(\phi)$ on CON and $(\subseteq \subseteq \Re$ respectively, and the map $\chi: \operatorname{EXT}\left(\phi_{0} \tau\right) \rightarrow H^{3}(\phi)$. We shall prove: the operations of $\tilde{H}^{2}(\phi)$ and $H^{2}(\phi)$ are simple, and their orbits are as in Theorem 5.3 ; the maps CON $\rightarrow$ CON $\rightarrow \mathfrak{G S N}$ $\rightarrow \operatorname{EXT}(\tau), \chi: \operatorname{EXT}\left(\phi_{0} \tau\right) \rightarrow H^{3}(\phi)$ are equivariant relative to the appropriate homomorphisms; the rectangle in (5.4) from $\mathbf{P}$ to $H^{3}(\phi)$ is commutative; sequence (5.9) is exact; the second assertion of Theorem 5.11 holds.

Consider $(G, \mu) \in \operatorname{EXT}\left(\phi_{0} \tau\right)$. By a weak structural cochain in $(G, \mu)$ we shall mean a triple $(f, v, t)$, where $f \in C^{2}(Q, K), v \in C^{1}(Q, G), t \in C^{1}\left[Q, A_{X}(K)\right]$, satisfying

$$
\mu v=1, \quad \phi_{\sharp} t=C_{N} v, \quad c_{K} f=\operatorname{dev} t, \quad \nu t=\tau .
$$

This differs from the concept of a "structural cochain in the normal homomorphism $\phi: K \rightarrow G$ " in the sense of $\$ 12$ of [CGE I] in that the condition $\phi f=\operatorname{dev} v$ has been dropped and the condition $\nu t=\tau$ added.

$S_{(\sigma, \mu)}$ will denote the set of all weak structural cochains in $(G, \mu)$. 
(7.2) For every $(G, \mu) \in \operatorname{EXT}\left(\phi_{0} \tau\right), S_{(G, \mu)}$ is nonempty.

Proof. Choose $v^{\prime} \in C^{1}(Q, G)$ and $t \in C^{1}\left[Q, A_{X}(K)\right]$ satisfying (7.1a) and (7.1d). Recall that $\bar{\nu}: A(N) \rightarrow A(N) / I(N)$ denotes the natural homomorphism. Then $\bar{\nu} \phi_{t} t=\phi_{0} \nu t=\phi_{0} \tau=\phi_{0} \tau \mu v^{\prime}=\bar{\nu} C_{N} v^{\prime}$, hence $\phi_{\sharp t} t-C_{N} v^{\prime} \subset I(N)$. Therefore there exists $d \in C^{1}(Q, N)$ such that $\phi_{t} t=c_{N} d+C_{N} v^{\prime}$. Let $v=d+v^{\prime}$. Since $\operatorname{dev} t$ $\subset I(K), f$ may be chosen to satisfy (7.1c). Q.E.D.

Given $h \in C^{1}(Q, K),(a, b) \in C^{2}(\phi),(f, v, t) \in S_{(G, \mu)}$, define

$$
\begin{aligned}
h+(f, v, t) & =\left(h_{t} \cdot f, \phi h+v, c_{K} h+t\right), \\
(a, b)+(f, v, t) & =(a+f, b+v, t) .
\end{aligned}
$$

It is easily verified that $C^{1}(Q, K)$ and $C^{2}(\phi)$ now act as groups of operators on $S_{(G, \mu)}$. Let $\bar{S}_{(G, \mu)}$ denote the set of all orbits in $S_{(G, \mu)}$ under $C^{1}(Q, K)$.

THEOREM 7.5. There exists one and only one operation of $C^{2}(\phi) / \tilde{B}^{2}(\phi)$ on $\bar{S}_{(G, \mu)}$ such that $S_{(G, \mu)} \rightarrow \bar{S}_{(G, \mu)}$ is equivariant relative to the natural homomorphism $\rho: C^{2}(\phi) \rightarrow C^{2}(\phi) / \widetilde{B}^{2}(\phi)$. The operation is simply transitive.

Proof. Let $\gamma: S_{(G, u)} \rightarrow \bar{S}_{(G, \mu)}$ denote the natural mapping. Consider $(a, b)$ $\in C^{2}(\phi)$ and $(f, v, t) \in S_{(G, \mu)}$. To establish the first assertion, it clearly suffices to show that the definition $\rho(a, b)+\gamma(f, v, t)=\gamma[(a, b)+(f, v, t)]$ is "independent of the choice of representatives."

So consider also $(c, d) \in \widetilde{B}^{2}(\phi)$ and $h \in C^{1}(Q, K)$; we must show that $(a, b)+(f, v, t)$ and $[(a, b)+(c, d)]+[h+(f, v, t)]$ lie in the same orbit under $C^{1}(Q, K)$.

Choose $g \in C^{1}\left(Q, Z_{K}\right)$ such that $(c, d)=D \epsilon_{K} g=(\delta g, \phi g)$. Then $g+h$ $\in C^{1}(Q, K)$, and $(g+h)+[(a, b)+(f, v, t)]=(g+h)+(a+f, b+v, t)=\left[(g+h)_{t}\right.$ $\left.\cdot(a+f), \phi(g+h)+b+v, c_{K}(g+h)+t\right]=\left[a+(g+h)_{t} \cdot f, \phi g+\phi h+b+v, c_{K} h+t\right]$ $=\left(a+\delta g+h_{t} \cdot f, b+\phi g+\phi h+v, c_{K} h+t\right)=\left(a+c+h_{t} \cdot f, b+d+\phi h+v, c_{K} h+t\right)$ $=(a+c, b+d)+\left(h_{t} \cdot f, \phi h+v, c_{K} h+t\right)=[(a, b)+(c, d)]+[h+(f, v, t)]$, completing the first portion of the proof.

Now consider any $(f, v, t)$ and $\left(f^{\prime}, v^{\prime}, t^{\prime}\right) \in S_{(G, \mu)} . \nu\left(t^{\prime}-t\right)=0$; hence we may choose $h \in C^{1}(Q, K)$ such that $t^{\prime}-t=c_{K} h$. Let $(a, b)=\left(h_{t} \cdot f-f^{\prime}, \phi h+v-v^{\prime}\right)$ $\in C^{2}(Q, K)+C^{1}(Q, N)$. Then $c_{K} a=c_{K}\left(h_{t} \cdot f\right)-c_{K} f^{\prime}=\operatorname{dev}\left(c_{K} h+t\right)-\operatorname{dev} t^{\prime}=0$, and $c_{N} b=c_{N} \phi h+C_{N} v-C_{N} v^{\prime}=\phi t\left(c_{K} h+t-t^{\prime}\right)=0$; therefore $(a, b) \in C^{2}(\phi)$. Now $\rho(a, b)+\gamma\left(f^{\prime}, v^{\prime}, t^{\prime}\right)=\gamma\left(a+f^{\prime}, b+v^{\prime}, t^{\prime}\right)=\gamma\left(h_{t} \cdot f, \phi h+v, c_{K} h+t\right)=\gamma[h+(f, v, t)]$ $=\gamma(f, v, t)$, showing that the new operation is transitive.

Suppose, finally, that $\rho(a, b)+\gamma(f, v, t)=\gamma(f, v, t)$. This means there exists $h \in C^{1}(Q, K)$ such that $(a+f, b+v, t)=h+(f, v, t)=\left(h_{t} \cdot f, \phi h+v\right.$, $\left.c_{K} h+t\right)$. From $t=c_{K} h+t$ we infer $h \in C^{1}\left(Q, Z_{K}\right)$; hence $(a+f, b+v)=(\delta h+f$, $\phi h+v)$, hence $(a, b)=(\delta h, \phi h)=D \epsilon_{K} h \in \widetilde{B}^{2}(\phi)$, hence $\rho(a, b)=0$; therefore the operation is simple. Q.E.D.

REMARK. If $R$ denotes the group of all $[(a, b), h] \in C^{2}(\phi)+C^{1}\left(Q, Z_{K}\right)$ such that $(a, b)+D \epsilon_{K} h=0$, it may be shown that $\left[C^{2}(\phi)+C^{1}(Q, K)\right] / R$ operates simply transitively on $S_{(G, \mu)}$. We shall not need this fact, however. 
Given $(f, v, t) \in S_{(G, \mu)}$, define

$$
\begin{gathered}
\delta(f, v, t)=\left(\delta_{t} f, \operatorname{dev} v-\phi f\right) \in C^{3}(Q, K)+C^{2}(Q, N) . \\
\delta\left[S_{(G, \mu)}\right] \subset Z^{3}(\phi) .
\end{gathered}
$$

Proof. By Theorem 5.17 of [CGE I] (derived from [CTAG II]), $\delta_{t} f$ $\in Z^{3}\left(Q, Z_{K}\right) . c_{N}(\operatorname{dev} v-\phi f)=\operatorname{dev}\left(C_{N} v\right)-\phi_{\# c_{K}} f=\operatorname{dev}\left(\phi_{\sharp t}\right)-\phi_{\#} \operatorname{dev} t=0$. Therefore $\delta(f, v, t) \in C^{3}(\phi)$, and $\pi_{K} D \delta(f, v, t)=0 . \pi_{N} D \delta(f, v, t)=\pi_{N} D\left(\delta_{t} f\right.$, $\operatorname{dev} v-\phi f)=\delta(\operatorname{dev} v-\phi f)+\phi\left(\delta_{t} f\right)=\delta(\operatorname{dev} v-\phi f)+\delta_{\phi \sharp t}(\phi f)=\delta_{\phi \neq t}(\operatorname{dev} v-\phi f$ $+\phi f)=\delta_{C_{N} v}(\operatorname{dev} v)=0$. Therefore $D \delta(f, v, t)=0$. Q.E.D.

(7.8) If $h \in C^{1}(Q, K)$ and $(f, v, t) \in S_{(G, \mu)}$, then $\delta[h+(f, v, t)]=\delta(f, v, t)$. ("Any two equivalent weak structural cochains have the same coboundary.")

Proof. By Lemma 5.16 of [CGE I], $\delta_{c_{K} h+t}\left(h_{t} \cdot f\right)=\delta_{t} f$. By (7.7), dev $v-\phi f$ $\in C^{2}\left(Q, Z_{N}\right)$. Hence, using formulae (4.8) and (4.5) of [CGE I], $\phi\left(h_{t} \cdot f\right)$ $=(\phi h)_{\phi_{\boldsymbol{f}} t} \cdot(\phi f)=(\phi h)_{C_{N} v} \cdot[(\phi f-\operatorname{dev} v)+\operatorname{dev} v]=(\phi f-\operatorname{dev} v)+(\phi h)_{c_{N^{v}} \cdot(\operatorname{dev} v)}$ $=\phi f-\operatorname{dev} v+\operatorname{dev}(\phi h+v)$. Therefore $\delta[h+(f, v, t)]=\left[\delta_{c_{K^{h+t}}}\left(h_{t} \cdot f\right), \operatorname{dev}\right.$ $\left.(\phi h+v)-\phi\left(h_{t} \cdot f\right)\right]=\left(\delta_{t} f, \operatorname{dev} v-\phi f\right)=\delta(f, v, t)$. Q.E.D.

Hence $\delta: S_{(G, \mu)} \rightarrow Z^{3}(\phi)$ induces a mapping $\delta: \bar{S}_{(G, \mu)} \rightarrow Z^{3}(\phi)$.

(7.9) $\delta: S_{(G, \mu)} \rightarrow Z^{3}(\phi)$ is equivariant rel. $D: C^{2}(\phi) \rightarrow Z^{3}(\phi)$.

Proof. $\delta[(a, b)+(f, v, t)]=\left[\delta_{t}(a+f), \operatorname{dev}(b+v)-\phi(a+f)\right]=\left(\delta a+\delta_{t} f\right.$, $\delta b-\phi a+\operatorname{dev} v-\phi f)=D(a, b)+\delta(f, v, t)$. Q.E.D.

Therefore, by (2.13),

(7.10) $\delta: \bar{S}_{(G, \mu)} \rightarrow Z^{3}(\phi)$ is equivariant rel. $D_{\sharp}: C^{2}(\phi) / \widetilde{B}^{2}(\phi) \rightarrow Z^{3}(\phi)$.

Applying (2.8) to (7.10), we find that $\delta\left[\bar{S}_{(\sigma, \mu)}\right]$ is a coset of

$$
D_{f}\left[C^{2}(\phi) / \tilde{B}^{2}(\phi)\right]=D\left[C^{2}(\phi)\right]
$$

in $Z^{3}(\phi)$. In other words, $\delta\left[S_{(G, \mu)}\right]$ is a coset of $B^{3}(\phi)$ in $Z^{3}(\phi)$. We define $\chi(G, \mu)=\delta\left[S_{(G, \mu)}\right] \in H^{3}(\phi)$. Clearly $\chi: \operatorname{EXT}\left(\phi_{0} \tau\right) \rightarrow H^{3}(\phi)$ is constant over each isomorphism-class; hence it induces a mapping

$$
\chi: \operatorname{EXT}\left(\phi_{0} \tau\right) \rightarrow H^{3}(\phi) .
$$

(7.11) In the sense of $\S 5$ of [CGE I], let $(f, t)$ be a structural cochain in the $Q$-kernel $\tau: Q \rightarrow A_{X}(K) / I(K)$, and let $(g, \phi \# t)$ be a structural cocycle in the $Q$ kernel $\phi_{0} \tau: Q \rightarrow A(N) / I(N)$, representing $\mathrm{e} \in \mathrm{EXT}\left(\phi_{0} \tau\right)$. Then $\left(\delta_{t} f, g-\phi f\right)$ $\in Z^{3}(\phi)$ represents $\chi \mathbf{e} \in \bar{H}^{3}(\phi)$.

Proof. Choose $(G, \mu)$ representing e. Then there exists $v \in C^{1}(Q, G)$ such that $\mu v=1, \operatorname{dev} v=g, C_{N} v=\phi_{\# t}$. Now $(f, v, t)$ is a weak stuctural cochain in $(G, \mu)$, and $\chi e$ is represented by $\delta(f, v, t)=\left(\delta_{t} f, g-\phi f\right)$. Q.E.D.

$(7.12) \chi: \operatorname{EXT}\left(\phi_{0} \tau\right) \rightarrow H^{3}(\phi)$ is equivariant rel. $\left(\epsilon_{N}\right)_{*}: H^{2}\left(Q, Z_{N}\right) \rightarrow H^{3}(\phi)$.

Proof. Given $a \in Z^{2}\left(Q, Z_{N}\right)$ and $e \in \operatorname{EXT}\left(\phi_{0} \tau\right)$. Choose a structural cochain $(f, t)$ in the $Q$-kernel $\tau: Q \rightarrow A_{X}(K) / I(K)$; then $e$ may be represented by a structural cocycle of the form $(g, \phi \sharp t)$, and the structural cocycle $(a+g, \phi \sharp t)$ 
represents $\rho a+e$. Therefore, by (7.11), $\chi(\rho a+e)=\rho\left(\delta_{t} f, a+g-\phi f\right)=\rho\left[\epsilon_{N}(a)\right.$ $\left.+\left(\delta_{t} f, g-\phi f\right)\right]=\rho \epsilon_{N}(a)+\rho\left(\delta_{t} f, g-\phi f\right)=\left(\epsilon_{N}\right)_{*} \rho(a)+\chi e$. Q.E.D.

(7.13) The rectangle in (5.4) from $\mathbf{P}$ to $H^{3}(\phi)$ is commutative.

Proof. Given a modular expansion $p=(G, \mu, \theta) \in \mathrm{P}$. Choose a structural cochain $(f, v, t)$ in the pseudo-module $(\phi, \theta)$, in the sense of [CGE I], $\S 10$. Then $(f, v, t) \in S_{(G, \mu)}$ and $\phi f=\operatorname{dev} v$. Therefore $\left(\epsilon_{K} i\right)_{*} \chi p=\left(\epsilon_{K} i\right)_{*} \rho\left(\delta_{t} f\right)$ $=\rho\left(\delta_{t} f, 0\right)=\rho\left(\delta_{t} f, \operatorname{dev} v-\phi f\right)=\chi(G, \mu)$. Q.E.D.

A structural cocycle in $(G, \mu) \in \operatorname{EXT}\left(\phi_{0} \tau\right)$ is an element $(f, v, t) \in S_{(\sigma, \mu)}$ whose coboundary is zero. This is the same thing as a structural cocycle in $\phi: K \rightarrow G$, in the sense of $\$ 12$ of [CGE I], satisfying the additional condition $\nu t=\tau$. Hence by $\$ 12$ of [CGE I],

(7.14) Given $(G, \mu) \in \operatorname{EXT}\left(\phi_{0} \tau\right)$. Then $(G, \mu)$ comes from an element of CON if and only if $(G, \mu)$ admits a structural cocycle.

It follows at once that (1.4), and hence (5.9), is exact.

Using $\$ 11$ of [CGE I], it is easy to show that $(G, \mu)$ comes from an element of $\mathrm{P}$ if and only if $(G, \mu)$ admits a weak structural cochain $(f, v, t)$ such that $\phi f=\operatorname{dev} v$. This yields a proof of the exactness of (5.10); but we prefer the proof given in $\$ 10$.

Proof OF TheOREM $5.11 \mathrm{~b}$. By the remark at the end of $\S 5$, we may assume that $\chi\left[\operatorname{EXT}\left(\phi_{0} \tau\right)\right]$ and $\left(\pi_{K}\right)_{*}^{-1}[\chi(\tau)]$ are nonempty. Then, by $(7.12)$ and the exactness of the second row of $(4.6)$, both are cosets of $\left(\epsilon_{N}\right)_{*} H^{2}\left(Q, Z_{N}\right)$ in $H^{3}(\phi)$. To show that they are the same coset, it suffices to establish

(7.15) If $\mathbf{e} \in \operatorname{EXT}\left(\phi_{0} \tau\right)$ then $\left(\pi_{K}\right) * \chi \mathbf{e}=\chi(\tau)$.

This, however, follows immediately from (7.11). Q.E.D.

We return to the consideration of a fixed $(G, \mu) \in \operatorname{EXT}\left(\phi_{0} \tau\right)$. Let $S_{(G, \mu)}^{0}$ and $\bar{S}_{(G, \mu)}^{0}$ denote the (possibly empty) kernels of $\delta: S_{(G, \mu)} \rightarrow Z^{3}(\phi)$ and $\delta: \bar{S}_{(G, \mu)}^{0} \rightarrow Z^{3}(\phi)$ respectively. We note that $\bar{S}_{(G, \mu)}^{0}$ may also be regarded as the set of all orbits in $S_{(G, \mu)}^{0}$ under $C^{1}(Q, K)$, which operates on $S_{(G, \mu)}^{0}$ because of (7.8).

Applying (2.5) to (7.10), we find that the orbits in $\bar{S}_{(G, \mu)}$ under $D^{-1}(0)$ $=\widetilde{H}^{2}(\phi)$ are the fibers of $\delta: \bar{S}_{(G, \mu)} \rightarrow Z^{3}(\phi)$. In particular, $\widetilde{H}^{2}(\phi)$ operates simply transitively on $\bar{S}_{(G, \mu)}^{0}$. But by $\S 12$ of [CGE I], $\bar{S}_{(G, \mu)}^{0}$ is in a natural 1-1 correspondence with $\operatorname{CON}_{(G, \mu)}$. We may say that $\widetilde{H}^{2}(\phi)$ operates simply on all of CON, the orbits being the fibers of CON $\rightarrow \operatorname{EXT}\left(\phi_{0} \tau\right)$.

If $(f, v, t) \in S_{(G, \mu)}^{0}$ represents the continuation $\bar{\phi}: E \rightarrow G$, then $(f, t)$ is a structural cocycle in the $Q$-kernel $\tau: Q \rightarrow A_{X}(K) / I(K)$, representing $(E, \mu \bar{\phi})$ $\in \operatorname{EXT}(\tau)$. Consequently, a comparison of (7.4) with formulae (10.8) and (5.11) of [CGE I] yields immediately

(7.16) $\mathrm{CON} \rightarrow \mathrm{CON} \rightarrow \mathrm{EXT}(\tau)$ is equivariant relative to

$$
H^{2}\left(Q, X \cap Z_{K}\right) \stackrel{\left(\epsilon_{K} i\right)_{\sharp}}{\longrightarrow} \tilde{H}^{2}(\phi) \stackrel{\left(\pi_{K}\right)_{*} \beta}{\longrightarrow} H^{2}\left(Q, Z_{K}\right) .
$$

We record for reference the corollary 
(7.17) $\mathrm{CON} \rightarrow \mathrm{EXT}(\tau)$ is equivariant rel. $i_{*}: H^{2}\left(Q, X \cap Z_{K}\right) \rightarrow H^{2}\left(Q, Z_{K}\right)$. Since any two isomorphic continuations are similar $(\S 3)$, the elements of $\operatorname{CON}_{(G, \mu)}$ divide into "similarity classes," which are the fibers of $\operatorname{CON}_{(G, \mu)}$ $\rightarrow \mathfrak{S} \mathfrak{S} \mathfrak{R}_{(G, \mu)}$.

(7.18) The similarity classes of $\mathrm{CON}_{(G, \mu)}$ are the orbits under the subgroup $\beta^{-1}(0)=B^{2}(\phi) / \widetilde{B}^{2}(\phi)$ of $\widetilde{H}^{2}(\phi)$.

Proof. Consider two elements $\bar{\phi}: E \rightarrow G$ and $\bar{\phi}^{\prime}: E^{\prime} \rightarrow G$ of $\operatorname{CON}_{(G, \mu)}$. Choose $t \in C^{1}\left[Q, A_{X}(K)\right]$ such that $\nu t=\tau$. We know that $\phi: E \rightarrow G$ and $\bar{\phi}^{\prime}: E^{\prime} \rightarrow G$ may be represented by structural cocycles of the form $(f, v, t)$ and $\left(f^{\prime}, v^{\prime}, t\right)$ respectively. Let $\gamma: S_{(G, \mu)}^{0} \rightarrow \bar{S}_{(G, \mu)}^{0}$ denote the natural mapping, and let $x=\gamma\left(f^{\prime}, v^{\prime}, t\right)$ $-\gamma(f, v, t) \in \widetilde{H}^{2}(\phi)$. We have to show that $\bar{\phi}: E \rightarrow G$ and $\bar{\phi}^{\prime}: E^{\prime} \rightarrow G$ are similar if and only if $\beta x=0$, i.e. if and only if $\left(f^{\prime}-f, v^{\prime}-v\right) \in B^{2}(\phi)$.

Choose $d \in C^{1}(Q, E)$ and $d^{\prime} \in C^{1}\left(Q, E^{\prime}\right)$ such that $\left(\operatorname{dev} d, \bar{\phi} d, C_{K} d\right)=(f, v, t)$ and $\left(\operatorname{dev} d^{\prime}, \bar{\phi} d^{\prime}, C_{K} d^{\prime}\right)=\left(f^{\prime}, v^{\prime}, t\right)$. In the presence of $(3.4 \mathrm{a})$, condition $(3.4 \mathrm{~b})$ holds automatically for $e \in K$, and is therefore equivalent to $\Phi^{\prime} W d=\left(c_{G} n_{0}\right) d \bar{\phi} d$, i.e. $\Phi^{\prime} W d=\left(c_{G} n_{0}\right) v$. By the identification $Z_{N}=C^{0}\left(Q, Z_{N}\right)$ and the definition of $\delta: C^{0}\left(Q, Z_{N}\right) \rightarrow C^{1}\left(Q, Z_{N}\right)$, we have, for each $n_{0} \in Z_{N}$,

$$
\delta n_{0}=\left(1-c_{G} n_{0}\right) v .
$$

Therefore $\Phi: E \rightarrow G$ and $\Phi^{\prime}: E^{\prime} \rightarrow G$ are similar if and only if there exist a homomorphism $W: E \rightarrow E^{\prime}$ and an element $n_{0} \in Z_{N}$ such that $W \mid K=1$ and

$$
v=\delta n_{0}+\Phi^{\prime} W d .
$$

By Lemma 5.22 of [CGE I], the formula $W d=h+d^{\prime}$ establishes a 1-1 correspondence between homomorphisms $W: E \rightarrow E^{\prime}$ satisfying

$$
W \mid K=1, \quad \mu \bar{\phi}^{\prime} W=\mu \bar{\phi},
$$

and cochains $h \in C^{1}\left(Q, Z_{K}\right)$ satisfying $f=h_{t} \cdot f^{\prime}\left(=\delta h+f^{\prime}\right)$. Therefore $\bar{\phi}: E \rightarrow G$ and $\Phi^{\prime}: E^{\prime} \rightarrow G$ are similar if and only if there exists $\left(h, n_{0}\right) \in C^{1}(\phi)$ such that $f=\delta h+f^{\prime}$ and $v=\delta n_{0}+\bar{\phi}^{\prime}\left(h+d^{\prime}\right)\left[=\delta n_{0}+\phi h+v^{\prime}\right]$. Therefore the continuations are similar if and only if there exists $\left(h, n_{0}\right) \in C^{1}(\phi)$ such that $\left(f-f^{\prime}, v-v^{\prime}\right)$ $=\left(\delta h, \delta n_{0}+\phi h\right)=D\left(h, n_{0}\right)$. Q.E.D.

Hence, by (2.12),

(7.22) There exists one and only one operation of $H^{2}(\phi)$ on $(\mathfrak{S} \mathfrak{N}$ such that $\mathrm{CON} \rightarrow \mathbb{S} \subseteq \mathfrak{N}$ is equivariant rel. $\beta: \widetilde{H}^{2}(\phi) \rightarrow H^{2}(\phi)$. The operation is simple, and the orbits are the fibers of $\mathbb{S} \mathfrak{S} \mathfrak{R} \rightarrow \mathrm{EXT}\left(\phi_{0} \tau\right)$.

By (2.13) and the second half of (7.16), $\mathfrak{\complement} \subseteq \mathfrak{R} \rightarrow \operatorname{EXT}(\tau)$ is equivariant rel. $\left(\pi_{K}\right)_{*}: H^{2}(\phi) \rightarrow H^{2}\left(Q, Z_{K}\right)$.

8. Differentiation; the graph. In this section we shall apply the results of [CGE I] and [CGE II] to obtain the simply transitive operation of $H^{2}(Q$, $\left.\phi Z_{K}\right)$ on $\mathrm{P}$ and a criterion for the existence of a modular expansion. We shall also show that $\mathrm{P} \rightarrow \mathrm{EXT}\left(\phi_{0} \tau\right)$ is equivariant rel. $j_{*}: H^{2}\left(Q, \phi Z_{K}\right) \rightarrow H^{2}(Q$, $Z_{N}$ ), and we shall prepare the ground for several of the proofs in later sections. 
In the present paper, our given data consist of the groups $Q, K, X$ and the homomorphism $\tau: Q \rightarrow A_{X}(K) / I(K)$. Now the groups $Q, N, \phi Z_{K}$ and the homomorphism $\phi_{0} \tau: Q \rightarrow A(N) / I(N)$ constitute a similar set of data, to which all our results may be applied. We call the system $\left(Q, N, \phi Z_{K}, \phi_{0} \tau\right)$ the $d e$ rivative of the system $(Q, K, X, \tau)$. By the derivative of any concept or statement formulated in terms of $(Q, K, X, \tau)$ we mean the same concept or statement as applied to $\left(Q, N, \phi Z_{K}, \phi_{0} \tau\right)$, and perhaps re-interpreted as a new concept or statement about $(Q, K, X, \tau)$. For instance, denoting differentiation by primes, we have

$$
\begin{aligned}
Q^{\prime} & =Q, \quad K^{\prime}=N, \quad X^{\prime}=\left(X \cap Z_{K}\right)^{\prime}=\phi Z_{K}, \quad \tau^{\prime}=\phi_{0} \tau, \\
\left(Z_{K}\right)^{\prime} & =Z_{N}, \quad \phi^{\prime}=\alpha, \quad\left(\phi Z_{K}\right)^{\prime}=\alpha\left(Z_{N}\right)=Z_{N} / \phi Z_{K}, \\
N^{\prime} & =N / \phi Z_{K}, \quad[\operatorname{EXT}(\tau)]^{\prime}=\operatorname{EXT}\left(\phi_{0} \tau\right), \quad i^{\prime}=j, \quad\left(\nabla_{1}\right)^{\prime}=\nabla_{2} .
\end{aligned}
$$

The derived system has certain special properties not generally shared by the original system. For example, $X^{\prime} \subset\left(Z_{K}\right)^{\prime}$. More significantly, since $\chi\left(\phi_{0} \tau\right)=j_{*} \phi_{*} \chi(\tau) \in j_{*} H^{3}\left(Q, \phi Z_{K}\right)$, we have $\left[\phi_{*} \chi(\tau)\right]^{\prime}=\alpha_{*} \chi\left(\phi_{0} \tau\right)=0$. By the derivative of the "empty" case of Theorem 5.11a (which we have not yet proved), this suggests that $P^{\prime}$ is always nonempty. It turns out, in fact, that the derived system admits a certain "natural" or "preferred" modular expan-

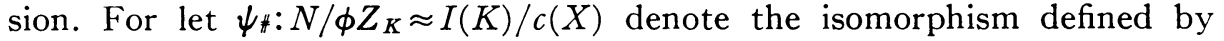
commutativity in

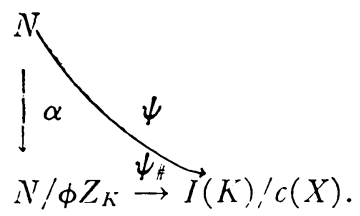

(See [CGE I, formulae 2.9, 2.10])

Then by Theorem 2.3 of [CGE II], the diagram

$$
\begin{array}{ll}
0 \rightarrow N / \phi Z_{K} & Q \longrightarrow 0 \\
\downarrow_{0} & \psi_{\star} \\
0 \rightarrow I(K) / c(X) \rightarrow A_{X}(K) / c(X) \stackrel{\omega}{\rightarrow} & A_{X}(K) / I(K) \rightarrow 0
\end{array}
$$

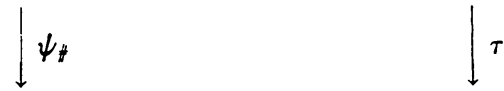

admits a completion

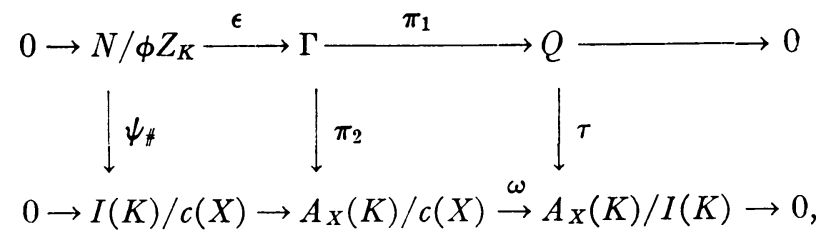


and any two such are isomorphic in one and only one way. We call $\left(\Gamma, \epsilon, \pi_{1}, \pi_{2}\right)$ the graph of the given system $(Q, K, X, \tau)$; it is the same as the graph of $\tau: Q \rightarrow A_{X}(K) / I(K)$ rel. $\omega: A_{X}(K) / c(X) \rightarrow A_{X}(K) / I(K)$, in the terminology of $\S 6$ of [CGE I], if we identify $N / \phi Z_{K}$ with $I(K) / c(X)$ via $\psi_{\# \text {. }}$

THEOREM 8.5. The homomorphism $\phi_{*} \pi_{2}: \Gamma \rightarrow A(N)$ is a modular structure on the normal homomorphism $\epsilon \alpha: N \rightarrow \Gamma$. P is in a natural 1-1 correspondence with the set of all extensions of the crossed module $\left(\epsilon \alpha, \phi_{*} \pi_{2}\right) ; \mathbf{P}$ is in a natural 1-1 correspondence with the set of all isomorphism-classes of extensions of the crossed module $\left(\epsilon \alpha, \phi_{*} \pi_{2}\right)$.

Proof. We noted at the end of $\S 2$ of [CGE I] that $\phi_{*}: A_{X}(K) / c(X) \rightarrow A(N)$ is a modular structure on the normal homomorphism $\psi: N \rightarrow A_{X}(K) / c(X)$. Therefore Theorem 5.4 of [CGE II], applied to the diagram

$$
0 \rightarrow N \quad Q \longrightarrow 0
$$

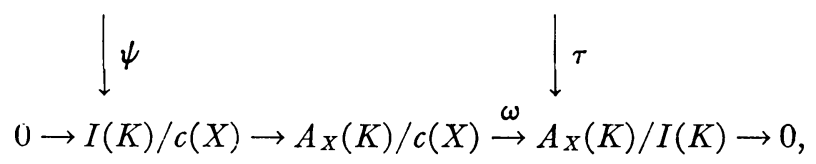

tells us: $\phi_{*} \pi_{2}: \Gamma \rightarrow A(N)$ is a modular structure on the normal homomorphism $\epsilon \alpha: N \rightarrow \Gamma$; the extensions of the crossed module $\left(\epsilon \alpha, \phi_{*} \pi_{2}\right)$ are in a natural 1-1 correspondence with those completions

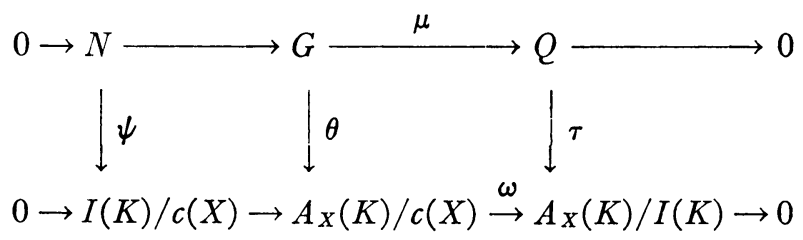

of (8.6) which satisfy the appropriate version of formula (5.3) of [CGE II], namely $\phi_{*} \theta=C_{N}$; ditto for isomorphism-classes. But we notice that by definition, an element of $\mathrm{P}$ is precisely a completion (8.7) satisfying $\phi_{*} \theta=C_{N}$; and isomorphism of "modular expansions" means the same as isomorphism of "completions of (8.6)." Q.E.D.

The derivative of $\omega: A_{X}(K) / c(X) \rightarrow A_{X}(K) / I(K)$ is

$$
\bar{\nu}: A_{\phi Z_{K}}(N) \rightarrow A_{\phi Z_{K}}(N) / I(N) .
$$

Now $\left(\phi_{0} \tau\right) \pi_{1}=\phi_{0} \omega \pi_{2}=\bar{\nu}\left(\phi_{*} \pi_{2}\right)$. Thus $\left(\Gamma, \pi_{1}, \pi_{2}\right)$ satisfies the derivative of condition (1.5); hence $\left(\Gamma, \pi_{1}, \pi_{2}\right) \in \mathrm{P}^{\prime}$.

The kernel of the normal homomorphism $\epsilon \alpha: N \rightarrow \Gamma$ is $\phi Z_{K}$. It is easily verified that the operation of $Q$ on $\phi Z_{K}$ resulting from the modular structure $\phi_{*} \pi_{2}: \Gamma \rightarrow A(N)$ coincides with the operation described in $\$ 4$ of the present paper. Therefore the cohomology groups $H^{n}(Q, X)$ of $\S \S 8$ and 9 of [CGE I] 
become, in the present application, $H^{n}\left(Q, \phi Z_{K}\right)$. Hence, combining Theorem 8.5 with [Theorem 8.5 and Corollary 9.5 of CGE I], we obtain

(8.8) $H^{2}\left(Q, \phi Z_{K}\right)$ operates in a natural way as a simply transitive transformation group on $\mathrm{P} . \mathrm{P}$ is nonempty if and only if the obstruction in $H^{3}\left(Q, \phi Z_{K}\right)$ of the crossed module $\left(\epsilon \alpha, \phi_{*} \pi_{2}\right)$ vanishes.

Theorem 8.5 focuses our attention on those continuations of the derived system which are extensions of the particular crossed module $\left(\epsilon \alpha, \phi_{*} \pi_{2}\right)$. We may say that $\mathrm{P}$ and $\mathbf{P}$ are the derivatives, respectively, of "a particular fiber of $\mathrm{CON} \rightarrow \mathrm{P}$ " and "a particular fiber of $\mathrm{CON} \rightarrow \mathrm{P}$." Then the operation of $H^{2}\left(Q, \phi Z_{K}\right)$ on $\mathrm{P}$ is, by construction, the derivative of the operation of $H^{2}\left(Q, X \cap Z_{K}\right)$ on any particular fiber of $\mathrm{CON} \rightarrow \mathrm{P}$. Therefore, by differentiating (7.17), we obtain

(8.9) $\mathrm{P} \rightarrow \operatorname{EXT}\left(\phi_{0} \tau\right)$ is equivariant rel. $j_{*}: H^{2}\left(Q, \phi Z_{K}\right) \rightarrow H^{2}\left(Q, Z_{N}\right)$.

9. Modified structural cochains. In this section we shall show that $\operatorname{EXT}(\tau) \rightarrow \mathrm{P}$ and $\chi: \mathrm{P} \rightarrow H^{3}\left(Q, X \cap Z_{K}\right)$ are equivariant relative to the corresponding homomorphisms; we shall also prove Theorem 5.11a.

DEFInition. A modified structural cochain is an ordered pair $(f, s)$, where $f \in C^{2}(Q, N)$ and $s \in C^{1}\left[Q, A_{X}(K) / c(X)\right]$, such that

$$
\omega s=\tau, \quad \psi f=\operatorname{dev} s .
$$

One easily verifies

(9.2) The passage from $(f, u)$ to $\left(f, \pi_{2} u\right)$ is a 1-1 mapping of the set of all structural cochains in the crossed module $\left(\epsilon \alpha, \phi_{*} \pi_{2}\right)$ (in the sense of [CGE I], $\S 9)$ onto the set of all "modified structural cochains."

We define "coboundary," "equivalence," etc. for modified structural cochains by composing the definitions in [CGE I], §§7-9 [as applied to the crossed module $\left.\left(\epsilon \alpha, \phi_{*} \pi_{2}\right)\right]$ with the 1-1 correspondence given by (9.2). We say that a modified structural cocycle $(f, s)$ represents a modular expansion $p \in \mathrm{P}$ if $(f, u)$ represents $e$, where $(f, u)$ corresponds to $(f, s)$ via $(9.2)$ and $e$ is the extension of $\left(\epsilon \alpha, \phi_{*} \pi_{2}\right)$ which corresponds to $p$ via Theorem 8.5. Then we find:

$$
\delta(f, s)=\delta_{\phi_{*}} f \in Z^{3}\left(Q, \phi Z_{K}\right) ;
$$

two modified structural cochains $(f, s)$ and $\left(f^{\prime}, s^{\prime}\right)$ are equivalent if and only if there exists $h \in C^{1}(Q, N)$ such that

$$
f^{\prime}=h_{\phi_{*}} \cdot f, \quad s^{\prime}=\psi h+s ;
$$

a modified structural cocycle $(f, s)$ represents a modular expansion $(G, \mu, \theta)$ if and only if there exists $v \in C^{1}(Q, G)$ such that

$$
\mu v=1, \quad \operatorname{dev} v=f, \quad \theta v=s ;
$$

the operation of $H^{2}\left(Q, \phi Z_{K}\right)$ on the set of equivalence classes of modified structural cocycles (and hence on $\mathbf{P}$ ) is induced by 


$$
a+(f, s)=(a+f, s)
$$

where $(f, s)$ is a modified structural cocycle and $a \in Z^{2}\left(Q, \phi Z_{K}\right)$.

Let $M$ denote the set of all modified structural cochains, and $S_{\tau}$ the set of all structural cochains in the $Q$-kernel $\tau: Q \rightarrow A_{X}(K) / I(K)$. With either symbol, the superscript 0 changes "cochain" to "cocycle."

(9.7) $\phi \times \lambda: C^{2}(Q, K)+C^{1}\left[Q, A_{X}(K)\right] \rightarrow C^{2}(Q, N)+C^{1}\left[Q, A_{X}(K) / c(X)\right]$ maps $S_{\tau}$ onto $M$ and $S_{\tau}^{0}$ into $M^{0}$.

Proof. Given $(g, t) \in S_{\tau}, \omega \lambda t=\nu t=\tau$, and $\psi \phi g=\lambda c_{K} g=\lambda \operatorname{dev} t=\operatorname{dev}(\lambda t)$; hence $(\phi g, \lambda t) \in M$. Conversely, given $(f, s) \in M$, choose $t \in C^{1}\left[Q, A_{X}(K)\right]$ such that $\lambda t=s$. Then $\psi f=\operatorname{dev} s=\lambda \operatorname{dev} t$; hence by Lemma 6.4 of [CGE I], there exists $g \in C^{2}(Q, K)$ such that $\phi g=f$ and $c_{K} g=\operatorname{dev} t . \nu t=\omega \lambda t=\omega s=\tau$; therefore $(g, t) \in S_{\tau}$ and $(\phi \times \lambda)(g, t)=(f, s)$. Finally, if $(g, t) \in S_{\tau}^{0}$, then $\delta[(\phi \times \lambda)(g, t)]=\delta_{\phi_{t} \lambda t} \phi g=\delta_{\phi_{t} t}(\phi g)=\phi\left(\delta_{t} g\right)=0$. Q.E.D.

(9.8) If $s \in S_{\tau}^{0}$ represents $e \in \mathrm{EXT}(\tau)$, and $p \in \mathrm{P}$ is the image of $e$ under the mapping $\operatorname{EXT}(\tau) \rightarrow \mathrm{P}$ of $\S 3$, then $(\phi \times \lambda)(s)$ represents $p$.

Proof. Let $(g, t)=s,(E, \rho)=e,(G, \mu, \theta)=p$ as usual. Choose $d \in C^{1}(Q, E)$ such that $\rho d=1, \operatorname{dev} d=g, C_{K} d=t$. Let $v=\bar{\phi} d \in C^{1}(Q, G)$, where $\Phi: E \rightarrow E / X$ $=G$ is the natural homomorphism, as in $\S 3$. Then $\mu v=\mu \Phi d=\rho d=1 ; \operatorname{dev} v$ $=\operatorname{dev}(\bar{\phi} d)=\bar{\phi} \operatorname{dev} d=\phi g ; \theta v=\theta \phi d=\lambda C_{K} d=\lambda t$, verifying the appropriate version of (9.5). Q.E.D.

Since $Z^{2}\left(Q, Z_{K}\right)$ and $Z^{2}\left(Q, \phi Z_{K}\right)$ operate on $S_{\tau}^{0}$ and $M^{0}$ respectively by means of the first coordinate in each case, it is obvious that $(\phi \times \lambda): S_{\tau}^{0} \rightarrow M^{0}$ is equivariant rel. $\phi: Z^{2}\left(Q, Z_{K}\right) \rightarrow Z^{2}\left(Q, \phi Z_{K}\right)$. Combining this with (9.8), we obtain

(9.9) $\operatorname{EXT}(\tau) \rightarrow \mathrm{P}$ is equivariant rel. $\phi_{*}: H^{2}\left(Q, Z_{K}\right) \rightarrow H^{2}\left(Q, \phi Z_{K}\right)$.

(9.10) Let $(g, t) \in S_{\tau}$ be such that $(\phi g, \lambda t) \in M^{0}$; let $(G, \mu, \theta)$ be a modular expansion represented by $(\phi g, \lambda t)$. Then $\chi(G, \mu, \theta)=\rho\left(\delta_{t} g\right) \in H^{3}\left(Q, X \cap Z_{K}\right)$.

Proof. Choose $v \in C^{1}(Q, G)$ satisfying $(9.5)$, with $(f, s)$ replaced by $(\phi g, \lambda t)$. Then $(g, v, t)$ is a structural cochain in the pseudo-module $\phi: K \rightarrow G, \theta: G$ $\rightarrow A_{X}(K) / c(X)$, in the sense of $\$ 10$ of [CGE I]. Q.E.D.

(9.11) $\chi: \mathrm{P} \rightarrow H^{3}\left(Q, X \cap Z_{K}\right)$ is equivariant rel. $\nabla_{1}: H^{2}\left(Q, \phi Z_{K}\right) \rightarrow H^{3}(Q$, $\left.X \cap Z_{K}\right)$.

Proof. Given $\phi a \in Z^{2}\left(Q, \phi Z_{K}\right)$ [where $\left.a \in C^{2}\left(Q, Z_{K}\right)\right]$ and $p \in \mathbf{P}$. Choose $(f, s) \in M^{0}$ representing the modular expansions in the isomorphism-class $\boldsymbol{p}$; by (9.7), choose $(g, t) \in S_{\tau}$ such that $(\phi g, \lambda t)=(f, s)$. Then the modular expansions in the isomorphism-class $\rho \phi a+p$ are represented by the modified structural cocycle $\phi a+(f, s)=[\phi(a+g), \lambda t]$. Therefore by (9.10), $\chi(\rho \phi a+p)$ $=\rho\left[\delta_{t}(a+g)\right]=\rho \delta a+\rho\left(\delta_{t} g\right)=\nabla_{1} \rho \phi a+\chi p$. Q.E.D.

(9.12) The obstruction of the crossed module $\left(\epsilon \alpha, \phi_{*} \pi_{2}\right)$ is equal to $\phi_{*} \chi(\tau)$ $\in H^{3}\left(Q, \phi Z_{K}\right)$.

Proof. Choose $(g, t) \in S_{\tau}$; then by (9.7), $(\phi g, \lambda t) \in M$. Therefore $\chi\left(\epsilon \alpha, \phi_{*} \pi_{2}\right)$ $=\rho \delta(\phi g, \lambda t)=\rho \delta_{\phi_{*} \lambda t}(\phi g)=\rho \delta_{\phi_{t} t}(\phi g)=\rho \phi\left(\delta_{t} g\right)=\phi_{*} \rho\left(\delta_{t} g\right)=\phi_{*} \chi(\tau)$. Q.E.D.

Combining (9.12) with $(8.8 \mathrm{~b})$, we obtain 
(9.13) There exists a modular expansion if and only if $\phi_{*} \chi(\tau)=0$ (cf. [APC]).

This proves the "empty" case of Theorem 5.11a. Assume now that $\chi(P)$ and $i_{*}^{-1}[\chi(\tau)]$ are nonempty. Then, by (9.11) and the exactness of the top row of (4.6), both are cosets of $\nabla_{1} H^{2}\left(Q, \phi Z_{K}\right)$ in $H^{3}\left(Q, X \cap Z_{K}\right)$. To show that they are the same coset, it suffices to establish

(9.14) If $p \in \mathrm{P}$, then $i_{*} \chi(p)=\chi(\tau)$ (cf. [APC]).

This, however, follows at once from (9.7) and (9.10).

10. Proofs by formal devices. In this section we conclude the proofs of Theorems 5.6 and 5.8 by showing that (5.10) is exact and that the rectangle in (5.4) from $\mathbf{P}$ to $H^{3}(\phi)$ is regular.

Let $\eta: \mathrm{P} \rightarrow \operatorname{EXT}\left(\phi_{0} \tau\right)$ denote the natural mapping.

(10.1) If $\boldsymbol{p} \in \mathbf{P}$ then $\left(\alpha \pi_{N}\right)_{*} \chi \eta \boldsymbol{p}=0$.

Proof. By (7.13), $\left(\alpha \pi_{N}\right)_{*} \chi \eta p=\left(\alpha \pi_{N}\right)_{*}\left(\epsilon_{K} i\right)_{*} \chi p=0$. Q.E.D.

(10.1) establishes half of the exactness of (5.10). To prove the other half, suppose $e \in \operatorname{EXT}\left(\phi_{0} \tau\right)$ and $\left(\alpha \pi_{N}\right)_{*} \chi e=0$. Then by (7.15) and the anticommutativity of (4.7) (with $n=3$ ), $\phi_{*} \chi(\tau)=\phi_{*}\left(\pi_{K}\right)_{*} \chi e=-\nabla_{2}\left(\alpha \pi_{N}\right)_{*} \chi e=0$. Therefore by (9.13), $\mathbf{P}$ is nonempty. Therefore by $(10.1),\left[\left(\alpha \pi_{N}\right)_{*} \chi \eta\right]^{-1}(0)$ is nonempty. But now (5.10) is exact by (2.7b).

Now suppose $\mathrm{e} \in \operatorname{EXT}\left(\phi_{0} \tau\right), h \in H^{3}\left(Q, X \cap Z_{K}\right)$, and $\chi \boldsymbol{e}=\left(\epsilon_{K} i\right)_{*} h$. Then by $(7.15), \phi_{*} \chi(\tau)=\phi_{*}\left(\pi_{K}\right)_{*} \chi e=\phi_{*}\left(\pi_{K}\right)_{*}\left(\epsilon_{K} i\right)_{*} h=\phi_{*} i_{*} h=0$. Therefore by (9.13), $\mathbf{P}$ is nonempty. The regularity of the rectangle from $P$ to $H^{3}(\phi)$ now follows from (7.13) and (2.15), because it is easily verified that the corresponding rectangle of homomorphisms is regular.

\section{PART III}

11. Automorphism groups. Given an extension (1.2) of the $Q$-kernel $\phi_{0} \tau: Q \rightarrow A(N) / I(N)$, let $A^{N}(G)$ denote the group of all automorphisms $W$ of $G$ satisfying

$$
W \mid N=1, \quad \mu W=\mu .
$$

$C^{1}(G, G)$, the set of all 1-cochains on $G$ in $G$, forms an additive group by addition of values in $G$. Elements of $C^{1}(G, G)$ may also be multiplied, by composition. $C^{1}(G, G)$ is then a "near-ring," satisfying all the axioms for a ring with identity except the commutativity of addition and the left distributive law $x(y+z)=x y+x z$.

(11.1) Given $d \in C^{1}(Q, G)$ such that $\mu d=1$, and $r \in C^{1}(Q, N)$. Then there exists at most one $W \in A^{N}(G)$ such that $W d=r+d$. There exists such $a W$ if and only if $r \in Z^{1}\left(Q, Z_{N}\right)$.

Proof. Transform the notation of Lemma (5.24) of [CGE I] as follows: $\left(f, t, Q, K, E, \rho, d, f^{\prime}, t^{\prime}, Q^{\prime}, K^{\prime}, E^{\prime}, \rho^{\prime}, d^{\prime}, \phi, \theta, r, \Phi\right) \rightarrow(f, t, Q, N, G, \mu, d, f, t$, $Q, N, G, \mu, d, 1,1, r, W)$. Then the lemma tells us: there exists at most one homomorphism $W: G \rightarrow G$ satisfying (11.1) and $W d=r+d$; there exists such 
a homomorphisn if and only if $f=r_{t} \cdot f$ and $t=c_{N} r+t$, where $f=\operatorname{dev} d$ and $t=C_{N} d$. But by the "five-lemma," any homomorphism $W: G \rightarrow G$ satisfying (11.2) is automatically an isomorphism and hence an element of $A^{N}(G)$; and by formula (4.6) of [CGE I], the conditions $f=r_{t} \cdot f$ and $c_{N} r=0$ are together equivalent to $r \in Z^{1}\left(Q, Z_{N}\right)$. Q.E.D.

It follows that for fixed $d \in C^{1}(Q, G)$ such that $\mu d=1$, the set of all $(r, W) \in Z^{1}\left(Q, Z_{N}\right) \times A^{N}(G)$ such that $W d=r+d$ is the graph of a function $\sigma_{d}: Z^{1}\left(Q, Z_{N}\right) \rightarrow A^{N}(G)$ which is $1-1$ onto. Given $n \in N, q \in Q$, and $r \in Z^{1}\left(Q, Z_{N}\right)$, we have $(r \mu+1)(n+d q)=r \mu(n+d q)+n+d q=r(\mu n+\mu d q)+n+d q=r q+n+d q$ $=n+r q+d q=n+(r+d) q=\left[\sigma_{d}(r)\right](n)+\left[\sigma_{d}(r)\right](d q)=\left[\sigma_{d}(r)\right](n+d q)$. Therefore $\sigma_{d}(r)=r \mu+1$. Hence $\sigma_{d}$ is independent of $d$, and we have proved that the function $\sigma: Z^{1}\left(Q, Z_{N}\right) \rightarrow A^{N}(G)$ defined by $\sigma(r)=r \mu+1$ is $1-1$ onto. But $(\sigma r)\left(\sigma r^{\prime}\right)=(r \mu+1)\left(r^{\prime} \mu+1\right)=r \mu\left(r^{\prime} \mu+1\right)+r^{\prime} \mu+1=r\left(\mu r^{\prime} \mu+\mu\right)+r^{\prime} \mu+1=r \mu+r^{\prime} \mu$ $+1=\left(r+r^{\prime}\right) \mu+1=\sigma\left(r+r^{\prime}\right)$. Therefore

(11.3) The formula $\sigma(r)=r \mu+1$ defines an isomorphism of the additive group $Z^{1}\left(Q, Z_{N}\right)$ onto the multiplicative group $A^{N}(G)$ (cf. [AEG]).

It follows, of course, that $A^{N}(G)$ is always commutative, and that up to isomorphism, $A^{N}(G)$ is independent of the particular extension $(G, \mu)$ of the $Q$-kernel $\phi_{0} \tau: Q \rightarrow A(N) / I(N)$.

We shall be interested in the subgroups $\sigma\left[\phi Z^{1}\left(Q, Z_{K}\right)\right] \subset \sigma\left[Z^{1}\left(Q, \phi Z_{K}\right)\right]$ $C A^{N}(G)$. We note:

$$
\begin{aligned}
A^{N}(G) / \sigma\left[Z^{1}\left(Q, \phi Z_{K}\right)\right] & \approx \alpha Z^{1}\left(Q, Z_{N}\right) \subset Z^{1}\left(Q, Z_{N} / \phi Z_{K}\right) \\
\sigma\left[Z^{1}\left(Q, \phi Z_{K}\right)\right] / \sigma\left[\phi Z^{1}\left(Q, Z_{K}\right)\right] & \approx H^{1}\left(Q, \phi Z_{K}\right) / \phi_{*} H^{1}\left(Q, Z_{K}\right) \\
& \approx \nabla_{1} H^{1}\left(Q, \phi Z_{K}\right) \subset H^{2}\left(Q, X \cap Z_{K}\right) .
\end{aligned}
$$

Applying the above to the particular extension of the $Q$-kernel $\alpha_{0} \phi_{0} \tau: Q$ $\rightarrow A(\alpha N) / I(\alpha N)$ given by the top row of (8.4), we obtain, as the "derivatives" of (11.3) and (11.5),

$$
\begin{aligned}
& \sigma^{\prime}: Z^{1}\left(Q, Z_{\alpha N}\right) \approx A^{\epsilon \alpha N}(\Gamma) \text { by } \sigma^{\prime}(r)=\epsilon r \pi_{1}+1 \\
& \sigma^{\prime}\left[Z^{1}\left(Q, Z_{N} / \phi Z_{K}\right)\right] / \sigma^{\prime}\left[\alpha Z^{1}\left(Q, Z_{N}\right)\right] \approx H^{1}\left(Q, Z_{N} / \phi Z_{K}\right) / \alpha_{*} H^{1}\left(Q, Z_{N}\right) \\
& \approx \nabla_{2} H^{1}\left(Q, Z_{N} / \phi Z_{K}\right) \subset H^{2}\left(Q, \phi Z_{K}\right) .
\end{aligned}
$$

For each $(G, \mu) \in \operatorname{EXT}\left(\phi_{0} \tau\right)$ we obtain, from (11.4),

$$
A^{N}(G) / \sigma\left[Z^{1}\left(Q, \phi Z_{K}\right)\right] \approx \sigma^{\prime} \alpha Z^{1}\left(Q, Z_{N}\right) \subset A^{\epsilon \alpha N}(\Gamma) .
$$

It may be verified that the homomorphism $\xi: A^{N}(G) \rightarrow A^{\epsilon \alpha N}(\Gamma)$ which induces (11.8) is characterized by

$$
(\xi W) \gamma=\epsilon \alpha(W g-g)+\gamma
$$

where $W \in A^{N}(G), \gamma \in \Gamma, g \in G$, and $\mu g=\pi_{1} \gamma$.

12. The operation of $Z^{1}\left(Q, Z_{N}\right)$ on $\operatorname{CON}$. Given $(G, \mu) \in \operatorname{EXT}\left(\phi_{0} \tau\right)$, let $\mathrm{CON}_{(G, \mu)}$ denote the counter-image of $(G, \mu)$ under the mapping $\mathrm{CON}$ 
$\rightarrow \operatorname{EXT}\left(\phi_{0} \tau\right)$. Given $(E, \bar{\phi}) \in \mathrm{CON}_{(\boldsymbol{G}, \mu)}$ and $W \in A^{N}(G)$, define $W(E, \bar{\phi})$ $=(E, W \Phi) \in \mathrm{CON}_{(G, \mu)}$. Then $A^{N}(G)$ operates as a left multiplicative transformation group on $\mathrm{CON}_{(\sigma, \mu)}$. It is readily verified that the operation is simple, and that the orbits are the fibers of $\mathrm{CON}_{(G, \mu)} \rightarrow \operatorname{EXT}(\tau)$. Combining this with (11.3), we obtain a simple operation of $Z^{1}\left(Q, Z_{N}\right)$ on $\operatorname{CON}_{(G, \mu)}$, with the same orbits. Since $Z^{1}\left(Q, Z_{N}\right)$ is independent of $(G, \mu)$, we may now say

(12.1) $Z^{1}\left(Q, Z_{N}\right)$ operates simply on $\mathrm{CON}$, the orbits being the fibers of $\mathrm{CON} \rightarrow \operatorname{EXT}\left(\phi_{0} \tau\right) \times \operatorname{EXT}(\tau)$.

(12.2) Given $(G, \mu) \in \operatorname{EXT}\left(\phi_{0} \tau\right)$ and $b \in Z^{1}\left(Q, Z_{N}\right)$. Let $(f, v, t)$ be a structural cocycle in $(G, \mu)(\S 7)$ representing $(E, \Phi) \in \operatorname{CON}_{(a, \mu)}$. Then $(f, b+v, t)$ is a structural cocycle in $(G, \mu)$ representing $b+(E, \bar{\phi})$.

Proof. By definition, $b+(E, \Phi)=[E,(b \mu+1) \Phi]$. Choose $d \in C^{1}(Q, E)$ such that $\mu \bar{\phi} d=1$ and $\left(\operatorname{dev} d, \bar{\phi} d, C_{K} d\right)=(f, v, t)$. Then $\left[\operatorname{dev} d,(b \mu+1) \bar{\phi} d, C_{K} d\right]$ $=\left(\operatorname{dev} d, b+\bar{\phi} d, C_{K} d\right)=(f, b+v, t)$. Therefore $(f, b+v, t)$ represents $b+(E, \bar{\phi})$; it follows automatically that $(f, b+v, t)$ is a structural cocycle. Q.E.D.

Comparison of (12.2) with (7.4) shows that $\mathrm{CON} \rightarrow \mathrm{CON}$ is equivariant rel. $\rho \epsilon_{N}: Z^{1}\left(Q, Z_{N}\right) \rightarrow \widetilde{H}^{2}(\phi)$.

13. The operations of $Z^{1}\left(Q, Z_{N} / \phi Z_{K}\right)$ on $\mathrm{P}$. In this section we shall describe two operations of $Z^{1}\left(Q, Z_{N} / \phi Z_{K}\right)$ on $\mathrm{P}$, which differ in sign. The "first" operation is the one referred to in Theorem 5.3 ; with respect to it, $\mathrm{CON} \rightarrow \mathrm{P}$ $\rightarrow \mathbf{P}$ is equivariant relative to

$$
\tilde{H}^{2}(\phi) \stackrel{\left(\alpha \pi_{N}\right)_{\boldsymbol{t}}}{\longrightarrow} Z^{1}\left(Q, Z_{N} / \phi Z_{K}\right) \stackrel{\nabla_{2} \rho}{\longrightarrow} H^{2}\left(Q, \phi Z_{K}\right) .
$$

Moreover, for each $(G, \mu) \in \operatorname{EXT}\left(\phi_{0} \tau\right)$, let $\mathrm{P}_{(G, \mu)}$ denote the counter-image of $(G, \mu)$ under the mapping $\mathrm{P} \rightarrow \operatorname{EXT}\left(\phi_{0} \tau\right)$. Then we shall describe a natural operation of $A^{N}(G)$ on $\mathrm{P}_{(G, \mu)}$ such that, using the "first" operation of $Z^{1}\left(Q, Z_{N} / \phi Z_{K}\right)$ on $\mathrm{P}_{(G, \mu)}$, the identity mapping $\mathrm{P}_{(G, \mu)} \rightarrow \mathrm{P}_{(G, \mu)}$ is equivariant relative to the composition $\alpha \sigma^{-1}: A^{N}(G) \rightarrow Z^{1}\left(Q, Z_{N} / \phi Z_{K}\right)$.

However, we shall show in $\S 15$ that the "second" operation is the "derivative" of the simply transitive operation (not yet established) of $Z^{1}\left(Q, \phi Z_{K}\right)$ on each fiber of $\mathrm{CON} \rightarrow \mathrm{P} \times \operatorname{EXT}(\tau)$.

In this section, to provide a clear basis for comparison, we shall describe both operations in terms of cochains. For the most part we shall consider a fixed $(G, \mu) \in \operatorname{EXT}\left(\phi_{0} \tau\right)$. We note that $\mathrm{P}_{(G, \mu)}$ may be identified with each of the following sets:

(a) the set of all modular structures $\theta: G \rightarrow A_{X}(K) / c(X)$ on the normal homomorphism $\phi: K \rightarrow G$ which satisfy (1.5);

(b) the set of all extensions of the crossed module $\epsilon \alpha: N \rightarrow \Gamma, \phi_{*} \pi_{2}: \Gamma$ $\rightarrow A(N)$ which determine $(G, \mu) \in \operatorname{EXT}\left(\phi_{0} \tau\right)(\S 8)$;

(c) the set of all equivalence classes of those frames $(u, s)$ [CGE I, §11] in the normal homomorphism $\phi: K \rightarrow G$ which satisfy $\omega s=\tau$.

The condition $\omega s=\tau$ will be implicitly assumed whenever we speak of "frames." 
Given a frame $(u, s)$, and $b \in C^{1}(Q, G)$, define

$$
b+(u, s)=(b+u, s) .
$$

(13.2) $b+(u, s)$ is a frame if and only if $b \in C^{1}\left(Q, Z_{N}\right)$ and $\alpha \delta b=0$.

Proof. Suppose $b+(u, s)$ is a frame. Then $1=\mu(b+u)=\mu b+1$, hence $\mu b=0$, i.e. $b \in C^{1}(Q, N) . c_{N} b+C_{N} u=C_{N}(b+u)=\phi_{*} s=C_{N} u$, hence $c_{N} b=0$, i.e. $b \in C^{1}\left(Q, Z_{N}\right) . \psi \delta b=\psi(\delta b+\operatorname{dev} u-\operatorname{dev} u)=\psi[\operatorname{dev}(b+u)-\operatorname{dev} u]=\operatorname{dev} s$ $-\operatorname{dev} s=0$; hence $\alpha \delta b=0$. The argument is clearly reversible. Q.E.D.

Let $\tilde{Z}^{1}\left(Q, Z_{N} / \phi Z_{K}\right)=C^{1}\left(Q, Z_{N}\right) \cap(\alpha \delta)^{-1}(0)$. It follows from (13.2) that (13.1) defines a simple operation of $\tilde{Z}^{1}\left(Q, Z_{N} / \phi Z_{K}\right)$ on the set of all frames [in $\phi: K \rightarrow G$, satisfying $\omega s=\tau]$. If the frames $(u, s)$ and $\left(u^{\prime}, s^{\prime}\right)$ are equivalent in the sense of [CGE I, §11] , and $b \in \tilde{Z}^{1}\left(Q, Z_{N} / \phi Z_{K}\right)$, then since $u-u^{\prime} \in C^{1}(Q, N)$, we have $\psi\left[(b+u)-\left(b+u^{\prime}\right)\right]=\psi\left[b+\left(u-u^{\prime}\right)-b\right]=\psi\left(u-u^{\prime}\right)=s-s^{\prime}$, hence $b+(u, s)$ and $b+\left(u^{\prime}, s^{\prime}\right)$ are equivalent. Therefore the operation of $\tilde{Z}^{1}\left(Q, Z_{N} / \phi Z_{K}\right)$ on the set of all frames induces an operation of

$$
\tilde{Z}^{1}\left(Q, Z_{N} / \phi Z_{K}\right)
$$

on the set of all equivalence classes of frames.

$b+(u, s)$ is equivalent to $(u, s)$ if and only if $s-s=\psi(b+u-u)$, i.e. if and only if $b \in C^{1}\left(Q, \phi Z_{K}\right)$. Since

$$
0 \rightarrow C^{1}\left(Q, \phi Z_{K}\right) \rightarrow \tilde{Z}^{1}\left(Q, Z_{N} / \phi Z_{K}\right) \stackrel{\alpha}{\rightarrow} Z^{1}\left(Q, Z_{N} / \phi Z_{K}\right) \rightarrow 0
$$

is exact, it follows that the operation of $\tilde{Z}^{1}\left(Q, Z_{N} / \phi Z_{K}\right)$ on the set of all equivalence classes of frames induces a simple operation of $Z^{1}\left(Q, Z_{N} / \phi Z_{K}\right)$ on the same set, and hence on $\mathrm{P}_{(\sigma, \mu)}$. This is our "first operation."

Let $T$ denote the set of all ordered pairs $(u, s)$ such that $u \in C^{1}(Q, G)$, $s \in C^{1}\left[Q, A_{X}(K) / c(X)\right]$, and $\mu u=1$. The concept of "equivalence" between two elements $(u, s)$ and $\left(u^{\prime}, s^{\prime}\right)$ of $T$, namely $s-s^{\prime}=\psi\left(u-u^{\prime}\right)$, makes sense whether the elements of $T$ are frames or not. If $(u, s)$ and $\left(u^{\prime}, s^{\prime}\right)$ are equivalent, it is easily verified that $\left(u^{\prime}, s^{\prime}\right)$ is a frame if and only if $(u, s)$ is a frame.

Given $(u, s) \in T$ and $b \in C^{1}(Q, N)$, define

$$
b+^{\prime}(u, s)=(u, \psi b+s) .
$$

(13.5) $b+^{\prime}(u, s)$ is equivalent to $-b+(u, s)$.

Proof. $(\psi b+s)-s=\psi[u-(-b+u)]$. Q.E.D.

Hence, by (13.2),

(13.6) If $(u, s)$ is a frame, then: $b+^{\prime}(u, s)$ is a frame if and only if $b \in C^{1}\left(Q, Z_{N}\right)$ and $\alpha \delta b=0$.

Using (13.5) at each step, it follows that (13.4), as well as (13.1), induces a simple operation of $Z^{1}\left(Q, Z_{N} / \phi Z_{K}\right)$ on $\mathrm{P}_{(\theta, \mu)}$, and that for each $h \in Z^{1}(Q$, $\left.Z_{N} / \phi Z_{K}\right)$ and each $\theta \in \mathrm{P}_{(G, \mu)}, h+^{\prime} \theta=-h+\theta$. The operation induced by (13.4) will be called the "second operation" and denoted with primes. 
(13.7) Using the first operation of $Z^{1}\left(Q, Z_{N} / \phi Z_{K}\right)$ on $\mathrm{P}_{(G, \mu)}, \operatorname{CON}_{(G, \mu)}$ $\rightarrow \mathrm{P}_{(G, \mu)}$ is equivariant rel. $\left(\alpha \pi_{N}\right) \neq: \tilde{H}^{2}(\phi) \rightarrow Z^{1}\left(Q, Z_{N} / \phi Z_{K}\right)$.

Proof. Let $F$ denote the set of all frames $(u, s)$ in $\phi: K \rightarrow G$ such that $\omega s=\tau$; let $\bar{F}$ denote the set of all equivalence classes of such frames. Recall from $\S 7$ that $S_{(G, \mu)}^{0}$ denotes the set of all structural cocycles in $(G, \mu)$, and that $\bar{S}_{(G, \mu)}^{0}$ denotes the set of all equivalence classes of such. Let $\xi: \operatorname{CON}_{(G, \mu)}$ $\rightarrow \mathrm{P}_{(G, \mu)}$ denote the natural mapping. By $\$ 12$ of [CGE I], the formula $\xi(f, v, t)=(v, \lambda t)$ defines a mapping $\xi: S_{(G, \mu)}^{0} \rightarrow F$, which induces a mapping $\xi: \bar{S}_{(G, \mu)}^{0} \rightarrow \bar{F}$ such that the diagram

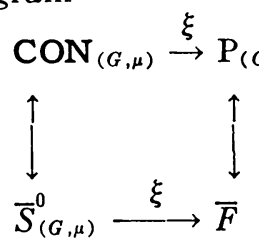

is commutative. It therefore suffices to show that $\xi: S_{(G, \mu)}^{0} \rightarrow F$ is equivariant rel. $\pi_{N}: Z^{2}(\phi) \rightarrow \tilde{Z}^{1}\left(Q, Z_{N} / \phi Z_{K}\right)$. But $\xi[(a, b)+(f, v, t)]=\xi(a+f, b+v, t)$ $=(b+v, \lambda t)=b+(v, \lambda t)=\pi_{N}(a, b)+\xi(f, v, t)$. Q.E.D.

The equivariance of $\mathrm{P} \rightarrow \mathrm{P}$ rel. $\nabla_{2} \rho: Z^{1}\left(Q, Z_{N} / \phi Z_{K}\right) \rightarrow H^{2}\left(Q, \phi Z_{K}\right)$ will be proved in $\$ 15$, where we shall also investigate the orbits.

Now consider $\theta \in \mathrm{P}_{(G, \mu)}$ and $W \in A^{N}(G)$. Then it is easily verified that $\theta W^{-1}: G \rightarrow A_{X}(K) / c(X)$ satisfies the required conditions, so that $\theta W^{-1}$ $\in \mathrm{P}_{(G, \mu)}$. Hence the formula $W \theta=\theta W^{-1}$ defines a left operation of $A^{N}(G)$ on $\mathrm{P}_{(G, \mu)}$. By definition of "congruence," the orbits under this operation are the fibers of $\mathrm{P}_{(G, \mu)} \rightarrow \mathfrak{B}$.

(13.9) Using the first operation of $Z^{1}\left(Q, Z_{N} / \phi Z_{K}\right)$ on $\mathrm{P}_{(G, \mu)}$, the identity map $\mathrm{P}_{(G, \mu)} \rightarrow \mathrm{P}_{(G, \mu)}$ is equivariant rel. $\alpha \sigma^{-1}: A^{N}(G) \rightarrow Z^{1}\left(Q, Z_{N} / \phi Z_{K}\right)$.

Proof. Given $W \in A^{N}(G)$ and $\theta \in \mathrm{P}_{(G, \mu)}$; let $r=\sigma^{-1}(W) \in Z^{1}\left(Q, Z_{N}\right)$, and choose a frame $(u, s)$ associated with $\theta$. Then $(W \theta)(r+u)=\theta W^{-1}(r+u)$ $=\theta r+\theta(-r \mu+1) u=\theta u=s$. Therefore $r+(u, s)=(r+u, s)$ is a frame associated with $W \theta$. Therefore $W \theta=\alpha r+\theta=\alpha \sigma^{-1} W+\theta$. Q.E.D.

14. The operation of $Z^{1}\left(Q, \phi Z_{K}\right)$ on $\mathrm{CON}$. We define the operation of $Z^{1}\left(Q, \phi Z_{K}\right)$ on $\mathrm{CON}$ by the condition that the identity mapping $\mathrm{CON} \rightarrow \mathrm{CON}$ shall be equivariant relative to the inclusion $j: Z^{1}\left(Q, \phi Z_{K}\right) \rightarrow Z^{1}\left(Q, Z_{N}\right)$.

Combining (13.7) with the last statement of $\$ 12$, we have

(14.1) Using the first operation of $Z^{1}\left(Q, Z_{N} / \phi Z_{K}\right)$ on $\mathrm{P}, \mathrm{CON} \rightarrow \mathrm{P}$ is equivariant rel. $\alpha: Z^{1}\left(Q, Z_{N}\right) \rightarrow Z^{1}\left(Q, Z_{N} / \phi Z_{K}\right)$.

Hence, by (12.1) and (2.5),

(14.2) The orbits in $\mathrm{CON}$ under $Z^{1}\left(Q, \phi Z_{K}\right)$ are the fibers of $\mathrm{CON} \rightarrow \mathrm{P}$ $\times \operatorname{EXT}\left(\phi_{0} \tau\right) \times \operatorname{EXT}(\tau)$, i.e. the fibers of $\mathrm{CON} \rightarrow \operatorname{P} \times \operatorname{EXT}(\tau)$.

(14.3) $\mathrm{CON} \rightarrow \mathrm{CON}$ is antivariant rel. $\nabla_{1} \rho: Z^{1}\left(Q, \phi Z_{K}\right) \rightarrow H^{2}\left(Q, X \cap Z_{K}\right)$.

Proof. This follows immediately from the following established facts about the upper left-hand rectangle of (5.4): the other three maps in the rectangle 
are equivariant; the two horizontal maps are identity maps; the corresponding rectangle of homomorphisms is anti-commutative.

15. The operations of $Z^{1}\left(Q, Z_{N} / \phi Z_{K}\right)$ on $\mathrm{P}$ (concluded). By (14.2), $Z^{1}\left(Q, \phi Z_{K}\right)$ operates simply transitively on each fiber of $\mathrm{CON} \rightarrow \operatorname{P} \times \operatorname{EXT}(\tau)$. Differentiating, we obtain a simply transitive operation of $Z^{1}\left(Q, Z_{N} / \phi Z_{K}\right)$ on each fiber of $\mathrm{P} \rightarrow \operatorname{EXT}\left(\phi_{0} \tau\right)$.

(15.1) For each $(G, \mu) \in \operatorname{EXT}\left(\phi_{0} \tau\right)$, the operation of $Z^{1}\left(Q, Z_{N} / \phi Z_{K}\right)$ on $\mathrm{P}_{(G, \mu)}$ obtained by differentiating the operation of $Z^{1}\left(Q, \phi Z_{K}\right)$ on $\mathrm{CON}$ coincides with the "second" operation, i.e. the one induced by (13.4).

Proof. Given $\theta \in \mathrm{P}_{(G, \mu)}$ and $\alpha b \in Z^{1}\left(Q, Z_{N} / \phi Z_{K}\right)$, where $b \in \tilde{Z}^{1}\left(Q, Z_{N} / \phi Z_{K}\right)$ $=C^{1}\left(Q, Z_{N}\right) \cap(\alpha \delta)^{-1}(0)$. Since $\omega \theta=\tau$, there exists one and only one homomorphism $\bar{\theta}: G \rightarrow \Gamma$ such that $\pi_{1} \bar{\theta}=\mu$ and $\pi_{2} \bar{\theta}=\theta ; \theta \in \mathrm{P}_{(G, \mu)}$ corresponds, via Theorem 8.5, to the extension $\bar{\theta}: G \rightarrow \Gamma$ of the crossed module $\epsilon \alpha: N \rightarrow \Gamma$, $\phi_{*} \pi_{2}: \Gamma \rightarrow A(N)$. Let $W=\sigma^{\prime}(\alpha b) \in A^{e \alpha N}(\Gamma)$. Then, in the sense of the "derivative," $\alpha b+\theta$ is the modular expansion corresponding to the extension $W \bar{\theta}: G$ $\rightarrow \Gamma$ of the crossed module $\left(\epsilon \alpha, \phi_{*} \pi_{2}\right)$. That is, in the sense of the "derivative," $\alpha b+\theta$ is the modular structure $\pi_{2} W \bar{\theta}: G \rightarrow A_{X}(K) / c(X)$ on the normal homomorphism $\phi: K \rightarrow G$.

Choose $u \in C^{1}(Q, G)$ such that $\mu u=1$. Then $(u, \theta u)$ is a frame associated with the modular structure $\theta: G \rightarrow A_{X}(K) / c(X)$. Therefore, in the sense of (13.4), $\alpha b+^{\prime} \theta$ is the modular structure associated with the frame $b+^{\prime}(u, \theta u)$ $=(u, \psi b+\theta u)$. But $\pi_{2} W \bar{\theta} u=\pi_{2}\left(\epsilon \alpha b \pi_{1}+1\right) \bar{\theta} u=\pi_{2} \epsilon \alpha b \pi_{1} \bar{\theta} u+\pi_{2} \bar{\theta} u=\psi \psi_{+\alpha} b \mu u+\theta u$ $=\psi b+\theta u$. Therefore $\alpha b+^{\prime} \theta=\pi_{2} W \bar{\theta}$. Q.E.D.

Therefore

(15.2) The orbits in $\mathrm{P}$ under the "second" operation by $Z^{1}\left(Q, Z_{N} / \phi Z_{K}\right)$ (and hence also under the "first" operation) are the fibers of $\mathrm{P} \rightarrow \operatorname{EXT}\left(\phi_{0} \tau\right)$.

Differentiating (14.3), and using the fact that the "first" and "second" operations differ in sign, we obtain

(15.3) Using the second (first) operation of $Z^{1}\left(Q, Z_{N} / \phi Z_{K}\right)$ on $\mathrm{P}, \mathrm{P} \rightarrow \mathrm{P}$ is antivariant (equivariant) rel. $\nabla_{2} \rho: Z^{1}\left(Q, Z_{N} / \phi Z_{K}\right) \rightarrow H^{2}\left(Q, \phi Z_{K}\right)$.

16. The operation of $\nabla_{2} H^{1}\left(Q, Z_{N} / \phi Z_{K}\right)$ on $\mathfrak{B}$. Henceforth we shall refer only to the first operation of $Z^{1}\left(Q, Z_{N} / \phi Z_{K}\right)$ on $\mathrm{P}$.

By (15.2), (15.3), and (2.5), the orbits in $\mathrm{P}$ under the kernel $\alpha Z^{1}\left(Q, Z_{N}\right)$ of $\nabla_{2} \rho: Z^{1}\left(Q, Z_{N} / \phi Z_{K}\right) \rightarrow H^{2}\left(Q, \phi Z_{K}\right)$ are the fibers of $\mathrm{P} \rightarrow \operatorname{EXT}\left(\phi_{0} \tau\right) \times \mathbf{P}$, i.e. the fibers of $\mathrm{P} \rightarrow \mathfrak{B}$. [This also follows from (13.9) and the remark preceding (13.9).] Therefore, by (2.12) and (15.2),

(16.1) There exists one and only one operation of $\nabla_{2} H^{1}\left(Q, Z_{N} / \phi Z_{K}\right)$ on $\mathfrak{B}$ such that $\mathrm{P} \rightarrow \mathfrak{B}$ is equivariant rel. $\nabla_{2} \rho: Z^{1}\left(Q, Z_{N} / \phi Z_{K}\right) \rightarrow \nabla_{2} H^{1}\left(Q, Z_{N} / \phi Z_{K}\right)$. The operation is simple, and the orbits are the fibers of $\mathfrak{B} \rightarrow \operatorname{EXT}\left(\phi_{0} \tau\right)$.

By (15.3) and (2.13), $\mathfrak{B} \rightarrow \mathbf{P}$ is equivariant relative to the inclusion $\nabla_{2} H^{1}\left(Q, Z_{N} / \phi Z_{K}\right) \rightarrow H^{2}\left(Q, \phi Z_{K}\right)$.

By $(13.7)$ and (16.1), $\mathrm{CON} \rightarrow \mathfrak{B}$ is equivariant rel. $\left(\nabla_{2} \rho\right)\left(\alpha \pi_{N}\right) \sharp: \widetilde{H}^{2}(\phi)$ $\rightarrow \nabla_{2} H^{1}\left(Q, Z_{N} / \phi Z_{K}\right)$. But $\left(\nabla_{2} \rho\right)\left(\alpha \pi_{N}\right)_{\#}=\left[\nabla_{2}\left(\alpha \pi_{N}\right)_{*}\right] \beta$, and $\operatorname{CON} \rightarrow(\mathfrak{N N}$ is 
equivariant rel. $\beta: \widetilde{H}^{2}(\phi) \rightarrow H^{2}(\phi)$. Therefore, by (2.13), $(\mathfrak{D} \mathfrak{R} \rightarrow \mathfrak{B}$ is equivariant rel. $\nabla_{2}\left(\alpha \pi_{N}\right)_{*}: H^{2}(\phi) \rightarrow \nabla_{2} H^{1}\left(Q, Z_{N} / \phi Z_{K}\right)$.

\section{BIBLIOGRAPHY}

[AEG] R. Baer, Automorphismen von Erweiterungsgruppen, Actualites Scientifiques et Industrielles, No. 205, Paris, 1935.

[APC] A. P. Cobbe, Some algebraic properties of crossed modules, Quart. J. Math. Oxford Ser. (2) vol. 2 (1951) pp. 269-285.

[CFB] R. L. Taylor, On the classification of fibre bundles, Ph.D. thesis, Oxford, 1950.

[CGE I] - Compound group extensions I, Trans. Amer. Math. Soc. vol. 75 (1953) pp. 106-135.

[CGE II] - Compound group extensions II, Trans. Amer. Math. Soc. vol. 75 (1953) pp. 304-310.

[CGE IV] M. Auslander, Compound group extensions IV, forthcoming.

[CNG] R. L. Taylor, Covering groups of nonconnected topological groups, Proc. Amer. Math. Soc. vol. 5 (1954) pp. 753-768.

[CTAG I] S. Eilenberg and S. MacLane, Cohomology theory in abstract groups I, Ann. of Math. vol. 48 (1947) pp. 51-58. 326-341.

[CTAG II] - Cohomology theory in abstract groups II, Ann. of Math. vol. 48 (1947) pp.

[CTAG III] S. MacLane, Cohomology theory in abstract groups III, Ann. of Math. vol. 50 (1949) pp. 736-761.

[E-S] S. Eilenberg and N. Steenrod, Foundations of algebraic topology, Princeton University Press, 1952.

[QKO I] A. P. Cobbe and R. L. Taylor, On Q-kernels with operators, I, forthcoming.

[QKO II] R. L. Taylor, On Q-kernels with operators II, forthcoming.

\section{Columbia University,}

NEW YORK, N. Y. 